\title{
On the Solution of the Steiner Tree NP-Hard Problem via Physarum BioNetwork
}

\author{
Marcello Caleffi, Member, IEEE, Ian F. Akyildiz, Fellow, IEEE, ACM, and Luigi Paura, Member, IEEE
}

\begin{abstract}
In the last several years, many algorithms trying to mimic biological processes have been proposed to enhance the performance of communication networks. However, the bio-inspired algorithms represent only the very first step toward the design of a smart adaptive communication network since: 1) they model only a limited set of the rules underlying the biological processes, thus, omitting fundamental functionalities; 2) they are executed on traditional computer architectures, thus, failing to achieve the intrinsic parallelism exhibited by biological processes. To overcome these issues, in this paper, the BioNetwork paradigm is proposed, a novel communication network paradigm in which the traditional network nodes are replaced by living organisms. The BioNetwork paradigm provides very attractive features over traditional network paradigms, such as efficiency, adaptivity, reliability, self-organization, and scalability. Moreover, it has a huge potential since it can be adopted in many different applications, such as health and military ones. In the paper, this potential is shown by proving that a BioNetwork can solve one of the most fundamental NP-hard problems in networks, i.e., the Steiner tree problem. To this aim, a BioNetwork constituted by a unicellular organism, the Physarum polycephalum slime mold, is designed. Throughout the paper, it is proven that a Physarum BioNetwork can solve the Steiner tree problem with an exponential convergence rate toward the optimal solution. The theoretical solutions are validated through a case study.
\end{abstract}

Index Terms-Biological networks, BioNetworks, Physarum polycephalum, Steiner tree.

\section{INTRODUCTION}

B IOLOGICAL organisms exhibit remarkable properties of self-organization, efficiency, and reliability when accomplishing the tasks needed to survive, as a direct consequence of

Manuscript received July 28, 2013; revised February 12, 2014; accepted March 25, 2014; approved by IEEE/ACM TRANSACTIONS ON NETWORKING Editor G. Xue. Date of publication June 09, 2014; date of current version August 14, 2015. This work was supported in part by the by the University of Naples Federico II under Award "Mobilità nell'Ambito delle Reti di Eccellenza: POR Campania FSE 2007-2013-Progetto MyOpenGov."

M. Caleffi was with the Broadband Wireless Networking Laboratory, School of Electrical and Computer Engineering, Georgia Institute of Technology, Atlanta, GA 30332 USA. He is now with the Department of Electrical Engineering and Information Technologies, University of Naples Federico II, 80125 Naples, Italy, and also with the Laboratorio Nazionale di Comunicazioni Multimediali, CNIT, 80126 Naples, Italy (e-mail: marcello.caleffi@unina.it).

I. F. Akyildiz is with the Broadband Wireless Networking Laboratory, School of Electrical and Computer Engineering, Georgia Institute of Technology, Atlanta, GA 30332 USA (e-mail: ian@ece.gatech.edu).

L. Paura is with the Department of Electrical Engineering and Information Technologies, University of Naples Federico II, 80125 Naples, Italy, and also with the Laboratorio Nazionale di Comunicazioni Multimediali, CNIT, 80126 Naples, Italy (e-mail: paura@unina.it).

Color versions of one or more of the figures in this paper are available online at http://ieeexplore.ieee.org.

Digital Object Identifier 10.1109/TNET.2014.2317911

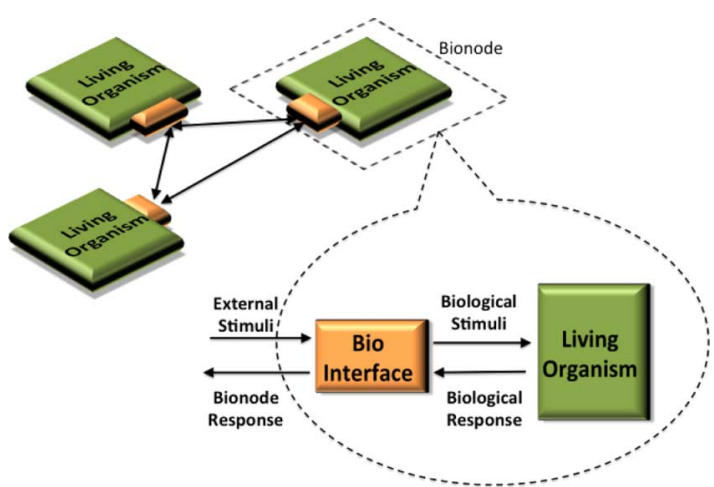

Fig. 1. BioNetwork model: Each bionode hosts one or more living organisms, and the responses of the living organisms to the external stimuli received through the bio-interfaces provide the network functionality.

the evolutionary process. For this, in the last 10 years, many algorithms and protocols mimicking the biological processes have been proposed to enhance the performance of communication networks [1].

Despite these efforts, the bio-inspired algorithms represent only the very first step toward the design of smart adaptive communication networks mainly for the following two reasons.

- Bio-inspired algorithms model only a limited set of the rules underlying the biological processes [2]. Hence, they omit undiscovered yet fundamental functionalities, thus failing to achieve the efficiency and the reliability exhibited by the original biological organisms.

- Bio-inspired algorithms are executed on traditional computer architectures, which do not offer the intrinsic parallelism exhibited by biological processes.

To overcome the aforementioned issues, in this paper we propose a novel communication network paradigm, referred to as BioNetwork paradigm, in which the network nodes host one or more biological organisms and the network operates according to the interactions among them. More specifically, in a BioNetwork, the traditional nodes are replaced by bionodes, i.e., by living organisms interfaced with each other through dedicated bio-interfaces, as shown in Fig. 1. The responses of the living organisms to the external stimuli received through the bio-interfaces provide the network functionalities.

Therefore, by exploiting a community of living organisms that interact with each other, the BioNetwork paradigm provides the following advantages over traditional algorithm-based network paradigms for building smart adaptive communication networks.

- Biological organisms are intrinsically efficient and reliable when accomplishing their processes by adapting 
themselves to the environmental conditions. BioNetworks inherit these properties since they are constituted by biological organisms.

- Biological processes are intrinsically autonomous and distributed, able to operate without the need of a centralized control or a global knowledge. Hence, BioNetworks are able to provide self-organizing and scalable communication services.

- Generally, several biological species coexist in the same ecosystem, either by competing for the same resources or by mutually benefiting from each other. Hence, BioNetworks constituted by different biological species are able to provide heterogeneous services with a fair resource allocation.

Clearly, the BioNetwork paradigm has a huge potential since it can be adopted in many different applications, due to the virtually uncountable degrees of freedom in selecting the biological species constituting a BioNetwork. Here, we disclose this potential by proving that a BioNetwork can solve one of the most fundamental NP-hard problems in networks, i.e., the Steiner tree problem. ${ }^{1}$ To this aim, we design a BioNetwork constituted by a unicellular organism, the Physarum polycephalum slime $\operatorname{mold}^{2}$ [3], and we analytically prove that a Physarum BioNetwork can solve the Steiner tree problem in graphs constituted by Physarum cycles ${ }^{3}$ with an exponential convergence rate toward the optimal solution.

The main contributions of this paper can be summarized as follows.

- We propose the novel BioNetwork paradigm, in which living organisms provide network functionalities.

- We design a BioNetwork for the Steiner tree problem constituted by Physarum cells.

- We develop a mathematical model for the evolution of a Physarum BioNetwork when a subset of the Physarum cells are artificially stimulated; this new mathematical model is able to describe the Physarum physiology when multiple food sources are available.

- We analytically prove, through the proposed model, that a Physarum BioNetwork can solve the Steiner tree problem in a graph constituted by Physarum cycles with an exponential convergence rate toward the optimal solution.

To the best of our knowledge, this is the first work that proposes and validates a BioNetwork, i.e., a network based on a community of living organisms that interact with each other.

The rest of the paper is organized as follows. In Section II, we discuss the related work. In Section III, we present the BioNetwork paradigm and discuss the challenges for BioNetwork design. In Section IV, we design a Physarum BioNet-

\footnotetext{
${ }^{1}$ Given a weighted undirected graph and a subset $S$ of vertices, the Steiner tree problem can be summarized as finding the minimum-cost tree spanning all the vertices in $S$. The formal definition of the problem and additional insights are given in Section IV-A.

${ }^{2}$ Physarum polycephalum is an ameboid unicellular organism, whose body contains a networks of protoplasmic veins that efficiently transport nutrients and chemical signals among different nuclei. Further details on Physarum polycephalum are given in Sections III-B and IV-B.

${ }^{3}$ The formal definition of Physarum cycle is given in Definition 14, Section V-B.
}

work for the Steiner tree problem and present the mathematical model describing the evolution of a Physarum BioNetwork. In Section V, through the aforementioned model, we analytically prove that a Physarum BioNetwork can solve the Steiner tree problem with an exponential convergence rate toward the optimal solution. Finally, Section VI provides the conclusions.

\section{RELATED WORK}

\section{A. BioNetwork Paradigm}

Very recently, researchers started to investigate the feasibility of unconventional computation paradigms, such as chemical, molecular, or biological computing. In [4], the feasibility of Physarum polycephalum is investigated as substrate for a novel computing architecture, and the study was followed by a research project funded by the European Commission under the Seventh Framework Programme (FP7) [5]. In [2], a biological organism, such as a cell culture, is adopted as an overlay structure to drive the functionalities of an underlying traditional communication network. The above-mentioned papers propose either a single biochip or a traditional two-tier network architecture with a centralized management driven by a biological process. Differently, in this paper, we propose a novel distributed network paradigm in which the traditional communication network is replaced by a community of living organisms that interact with each other for providing communication services.

\section{B. Physarum Polycephalum}

Physarum physiology has been widely studied for more than 50 years, and in the following, we focus on the works related to network aspects. In [6], the authors conduct an experimental study of the Physarum vein network. Their statistical analysis shows that the network geometry exhibits two remarkable properties: a short total-length and a tolerance toward accidental network disconnection. In [7], the authors present a mathematical model for the physiological mechanism underlying the Physarum vein network shaping when two food sources are available. Then, they successfully apply a bio-inspired algorithm based on such a model to the shortest-path problem for road navigation. In [8], the authors further investigate the same model in terms of equilibrium points, while in [9], the authors mathematically prove that the bio-inspired algorithm proposed in [7] converges to the optimal shortest-path solution with an exponential convergence rate. All the above-mentioned papers limit their attention to either experimental studies or the simple shortest-path problem. Differently, in this paper, we focus on analytically proving that a BioNetwork constituted by Physarum cells can solve one of the most fundamental problems in networks, i.e., the Steiner tree problem. Finally, in [10], a heuristic bio-inspired algorithm is proposed for the Steiner tree problem, which is a slight modification of the already-proposed Physarum-inspired algorithm for the shortest-path problem [7]. The algorithm fails very often in finding the minimum-length Steiner tree. Differently, in our paper, we propose a BioNetwork constituted by Physarum cells, and we analytically prove that a Physarum BioNetwork can solve the Steiner tree problem in graphs constituted by Physarum cycles with an exponential convergence rate toward the optimal solution. In addition, 


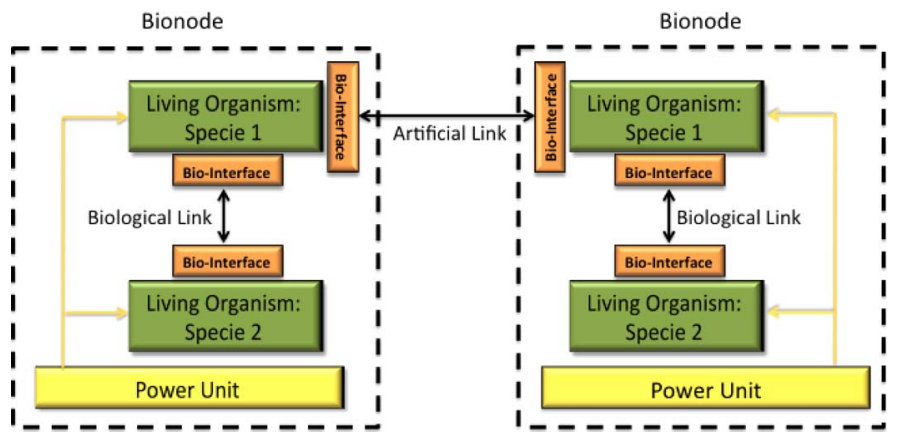

Fig. 2. BioNetwork example: Each bionode hosts two biological organisms belonging to different species, which interact through biological links. Artificial links provide connections among biological organisms hosted in different bionodes.

our analysis is carried out by developing a new mathematical model for the Physarum physiology in presence of multiple food sources.

\section{BIONETWORK PARADIGM}

Here, we first present the BioNetwork paradigm in Section III-A. Then, in Section III-B, we discuss the challenges for designing a network architecture based on the BioNetwork paradigm.

\section{A. BioNetwork Paradigm}

Definition 1: A BioNetwork is a communication network in which the network nodes, referred to as bionodes, host one or more biological organisms, and the network operates according to the interactions among them.

Hence, a BioNetwork is a community of living organisms that interact with each other through biological or artificial connections ${ }^{4}$ for providing communication services, as shown in Fig. 2.

BioNetworks can rely on one or more biological species, resulting in different levels of complexity, which could range from a network constituted by a single species of unicellular organisms to an ecosystem of several biological species that interact with each other. The simplest bionode architecture includes the following components, as shown in Fig. 2.

- Processing unit: The processing unit is the biological organism or, more generally, one of its biological processes. The status of the biological process represents the state register of the processing unit, and its evolution over time represents the transition function of the processing unit.

- Bio-interface unit: The bio-interface unit detects and induces changes in the biological process. It represents an input/output device for the biological organism, thus implementing a feedback system between the biological process and the other elements of the BioNetwork.

- Power unit: The power unit is the energy source of the biological organism, and it can vary from a simple light emission for photo-autotrophic organisms to a more complex organic molecule dispenser for heterotrophic organisms.

\footnotetext{
${ }^{4}$ The internode communications can rely on artificial connections, such as optical or electromagnetic links, since they provide high-rate long-range communications. Differently, intranode communications can rely on either biological or artificial connections.
}

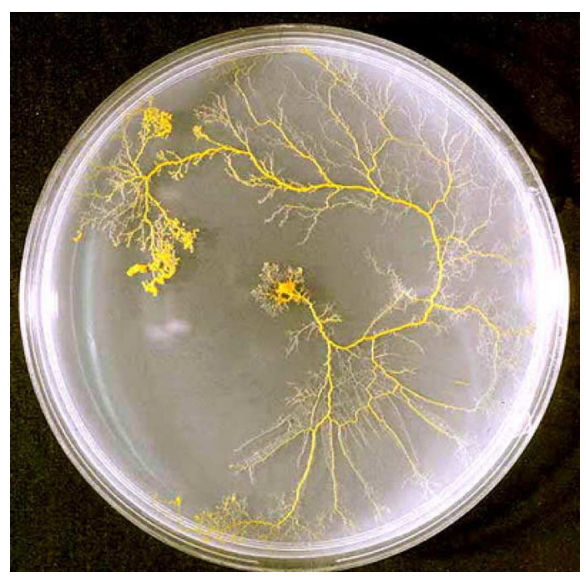

Fig. 3. Physarum polycephalum slime mold: The vein network connecting the nuclei is induced by a protoplasmic flow caused by the hydrostatic pressure generated by the rhythmic contractions of actin-myosin fibers.

\section{B. Challenges}

The design challenges of a BioNetwork are completely different from those of a traditional communication network. In fact, in a traditional network, the key issue for a protocol designer is to explicitly take into account all the possible scenarios to make the protocol adaptive. Differently, the key issue for a BioNetwork designer is to select, among the biological organisms available in nature, the most efficient and reliable in providing the desired network functionality for a specific operative environment.

More specifically, a candidate biological organism to be used in a BioNetwork should exhibit the following three properties.

- Effectiveness: The network functionality has to be directly mapped on a biological process underlying the physiology of the selected organism. ${ }^{5}$

- Controllability: To realize a feedback system between the biological organism and the other elements of the BioNetwork, artificial or biological stimuli able to detect and to induce changes on the biological process must be available.

- Survivability: The biological organism must survive in the operative network conditions, and its lifetime has to match with the network functionality timescale.

A very attractive candidate for the BioNetwork paradigm is the Physarum polycephalum slime mold [3]. In fact, it exhibits all the aforementioned properties. More specifically:

- Physarum effectiveness: Physarum polycephalum is an ameboid unicellular organism, whose body, during the plasmodium vegetative phase, contains a networks of protoplasmic veins that efficiently transport nutrients and chemical signals among different nuclei, as shown in Fig. 3. Very recently, the researchers discovered that the geometry of the vein network meets remarkable characteristics in presence of multiple food sources, such as short network length, adaptability toward environmental changes, and reliability toward accidental disconnections [6]. Moreover, the researchers found out that Physarum can solve the shortest-path problem when the two food sources are provided [8]. Thus, it is possible

${ }^{5}$ Clearly, biological processes needed by the organism to survive are the best candidates in terms of efficiency. 
to directly map network-layer functionalities to the biological process underlying the Physarum vein network shaping.

- Physarum controllability: The physiological mechanism underlying the Physarum vein network shaping can be controlled through external stimuli, both positive (glucose, warm) and negative (blue light, salt, cold). Very recently, some of these artificial stimuli are exploited to design and implement a chip based on a Physarum cell and interfaced with a hexapod robot [11]. The Physarum chip receives external inputs through light stimuli, and the protoplasmic flow oscillations, detected through a CCD camera, drive the robot movement.

- Physarum survivability: Physarum is able to survive in a wide range of environmental conditions, including the adverse ones through its sclerotium vegetative phase. Moreover, the researchers already successfully implemented the Physarum-based chips.

Stemming from these considerations, in Section IV, we design a BioNetwork constituted by the Physarum cells, and in Section V, we prove that it can solve the Steiner tree problem in a graph constituted by Physarum cycles with an exponential convergence rate toward the optimal solution.

\section{Physarum Bionetwork: Preliminaries and DEFINITIONS}

After a brief introduction of the Steiner tree problem in Section IV-A, we describe the proposed Physarum BioNetwork for the Steiner tree problem in Section IV-B. Then, in Section IV-C, we derive the mathematical model for the evolution of the Physarum BioNetwork, and we justify its formulation in terms of Physarum polycephalum physiology.

\section{A. Steiner Tree Problem}

We model a communication network with a connected undirected graph $G=(V, E)$, where a vertex $v_{i} \in V$ $=\left\{v_{1}, \ldots, v_{n}\right\}$ denotes a node, and an edge $e_{i j} \in E$ denotes the presence of a link 6 between the vertices $v_{i}$ and $v_{j}$. A graph $G^{\prime}=\left(V, E^{\prime}\right)$ is defined as subgraph of $G$ if $E^{\prime} \subseteq E$. We denote with $L: E \rightarrow \mathbb{R}^{+}$a function that assigns a length (cost) to each edge (link), and since $G$ is undirected, it follows $l_{i j} \triangleq L\left(e_{i j}\right)=L\left(e_{j i}\right) \triangleq l_{j i}$. Accordingly, the length $L\left(E^{\prime}\right)$ of a subgraph $G^{\prime}=\left(V, E^{\prime}\right) \subseteq G$ is defined as $L\left(E^{\prime}\right)=\sum_{e_{i j} \in E^{\prime}} L\left(e_{i j}\right)$.

Definition 2 (Simple Path): Given a graph $G$ and two arbitrary vertices $v_{i}, v_{j} \in V$, a simple path $P_{i j}=\left(V, E_{P_{i j}}\right) \subseteq G$ is a subgraph of $\mathrm{G}$ with

$$
E_{P_{i j}}=\left\{e_{k_{0} k_{1}}, e_{k_{1} k_{2}}, \ldots, e_{k_{N-1} k_{N}}\right\}
$$

where $k_{0}=i, k_{N}=j, i \neq j$, and $k_{m} \neq k_{l} \forall m, l$.

Definition 3 (Cycle): Given a graph $G$, a cycle $C$ $=\left(V, E_{C}\right) \subseteq G$ is a subgraph of $\mathrm{G}$ with

$$
E_{C}=\left\{e_{k_{0} k_{1}}, e_{k_{1} k_{2}}, \ldots, e_{k_{N-1} k_{N}}\right\}
$$

where $k_{0}=k_{N}$. A simple cycle $C$ is a cycle where $k_{m} \neq$ $k_{l} \forall e_{k_{m} k_{m+1}}, e_{k_{l} k_{l+1}} \in E_{C}, m, l \neq 0$.

\footnotetext{
${ }^{6}$ Without loss of generality, we assume throughout the paper that at most one link exists between each pair of nodes since our results can be easily extended to the case of multiple links by simply introducing fictitious nodes.
}

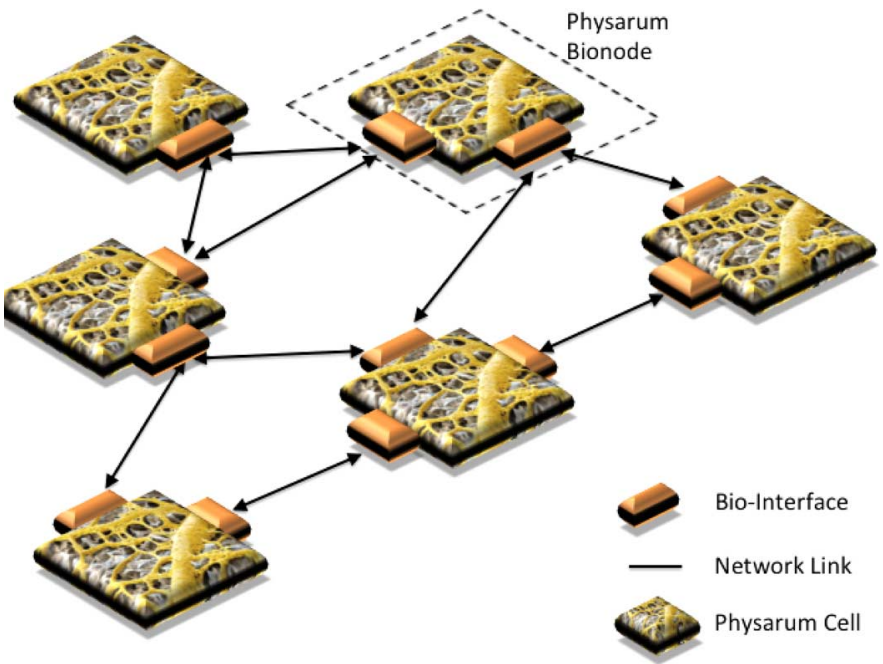

Fig. 4. Physarum BioNetwork: Each node hosts a Physarum cell enclosed in a chip, and the interactions among different cells are provided through bio-interfaces able to map network packets to actin-myosin fibers contractions.

Definition 4 (Tree): Given a graph $G$, a tree in $G$ is a connected subgraph of $G$ with no simple cycles.

Definition 5 (Steiner Tree): Given a graph $G$ and an arbitrary set $S \subseteq V$ of vertices referred to as Steiner vertices, a Steiner tree $\mathcal{T}=\left(V, E_{\mathcal{T}}\right) \subseteq G$ is a tree in $G$ that spans all the vertices in $S$.

Definition 6 (Direct Path): Given a graph $G$ and two arbitrary Steiner vertices $v_{i}, v_{j} \in S$, a direct path $P_{i j}^{d}=\left(V, E_{P_{i j}^{d}}\right) \subseteq G$ is a simple path in $G$ where $v_{k_{l}} \notin S \forall e_{k_{l} k_{l+1}} \in E_{P_{i j}^{d}}, l^{\prime} \neq 0$.

Similarly, an indirect path $P_{i j}^{i}=\left(V, E_{P_{i j}^{i}}\right)$ is a simple path where $\exists e_{k_{l} k_{l+1}} \in E_{P_{i j}^{i}}, l \neq 0: v_{k_{l}} \in S$.

Definition 7 (Minimum-Length Steiner Tree): Given a graph $G$, a set $S$ of Steiner vertices, and a length function $L: E$ $\rightarrow \mathbb{R}^{+}$, the minimum-length Steiner tree $\mathcal{T}_{\mathrm{opt}}=\left(V, E_{\mathcal{T}_{\mathrm{opt}}}\right)$ is the Steiner tree such that $L\left(E_{\mathcal{T}_{\text {opt }}}\right)=\min _{\mathcal{T} \subseteq G} L\left(E_{\mathcal{T}}\right)$

Definition 8 (Steiner Tree Problem in Graphs): Given a graph $G$, a set $S$ of Steiner vertices, and a length function $L: E$ $\rightarrow \mathbb{R}^{+}$, the problem is to find minimum-length Steiner tree $\mathcal{T}_{\text {opt }}$.

As generally known, the Steiner tree problem in graphs is a classical NP-hard problem [12] since the corresponding decision problem-i.e., determining whether a Steiner tree of total length at most $k$ exists for a given graph — was one of Karp's 21 NP-complete problems [13]. Steiner trees have been applied in many different areas, such as communication networks, circuit design, and computing, where many papers are published in the last decade.

\section{B. Physarum BioNetwork: Overview}

As mentioned in Section III-B, Physarum is an ameboid unicellular organism, whose body, during the plasmodium vegetative phase, contains a network of protoplasmic veins that efficiently transport nutrients and chemical signals among different nuclei, as shown in Fig. 3.

The Physarum vein network is induced by a protoplasmic flow caused by the hydrostatic pressure generated by rhythmic contractions of actin-myosin fibers. When a nutrient source is available, the actin-myosin fibers closer to the nutrient sources 


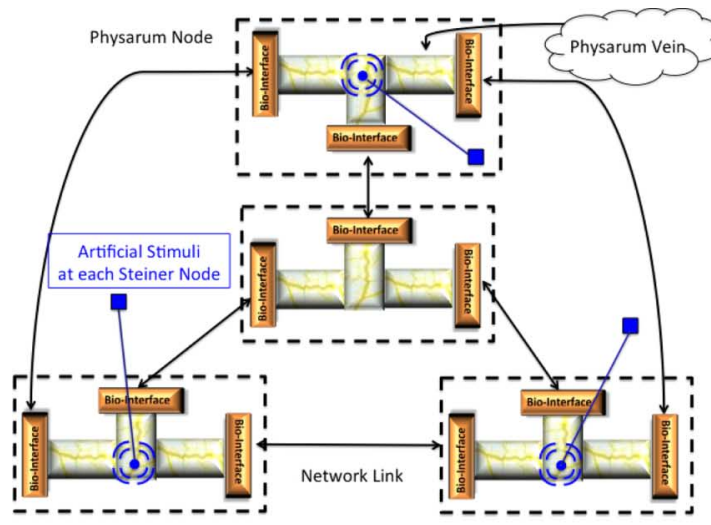

(a)

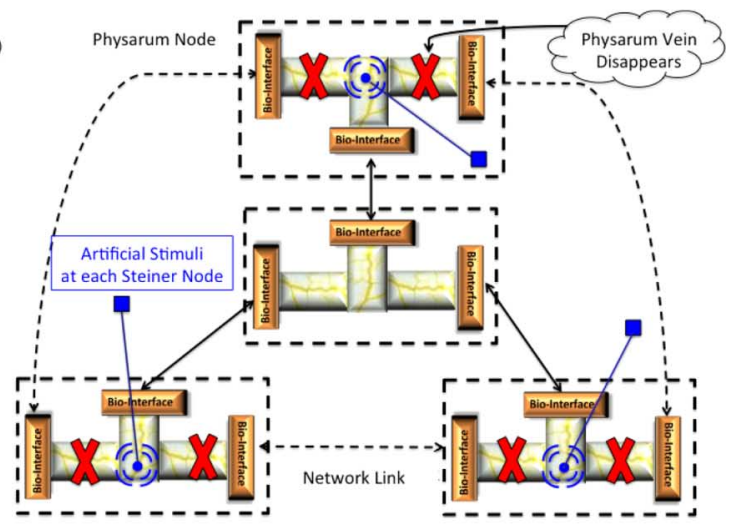

(b)

Fig. 5. Four-node Physarum BioNetwork evolution in presence of three Steiner nodes. (a) Time $t=0$. (b) Time $t=0+\delta$.

contract, thus generating the protoplasmic flow. It has been observed that the evolution of the vein network follows simple rules: 1) veins not connected to food sources tend to disappear; and 2) when multiple veins connect two food sources, the longer veins tend to disappear. We refer the reader to [8] for further details about the Physarum physiology underlying the vein network shaping.

According to Definition 1, a Physarum BioNetwork is a network constituted by Physarum cell connected to each other through network links, as shown in Fig. 4. The reception of a network packet through a link enables the bio-interface to artificially induce, inhibit, or modify the rhythmic contractions of actin-myosin fibers, e.g., through the release of calcium ions [14]. Similarly, specific patterns of actin-myosin rhythmic contractions are detected through the bio-interface and mapped in a network packet sent through the network link. In other words, all the Physarum cells (actually, all the nuclei contained in each Physarum cell) are connected through virtual protoplasmic veins, i.e., a pair of bio-interfaces and a network link. Each cell propagates the incoming virtual protoplasmic flow according to its internal vein network, and, in turn, its internal vein network evolves according to the incoming protoplasmic flow.

Hence, in our Physarum BioNetwork, the artificial stimuli drive the Physarum physiology to build an adaptive network in which the Steiner nodes are connected through the minimum-length Steiner tree. This mechanism is shown in Fig. 5 with a simple example: a four-node network with three Steiner nodes. The Steiner nodes are artificially stimulated with positive feedbacks to induce rhythmic contractions in the actin-myosin fibers [Fig. 5(a)]. The induced contractions generate a protoplasmic flow that is propagated, through the virtual veins, to every Physarum cell.

Consequently, at each cell, the biological process underlying the Physarum vein network shaping evolves according to the incoming protoplasmic flow. Eventually, the virtual veins that survive, i.e., the network links propagating virtual protoplasmic flow through network packets, form the minimum-length Steiner tree [Fig. 5(b)], as analytically proven in Section V.

\section{Physarum BioNetwork: Mathematical Model}

Stemming from the notation introduced in Section IV-A, in the following, a vertex $v_{i} \in V$ denotes a Physarum cell, and the set $S \subseteq V$ of Steiner vertices denotes the Physarum cells stimulated to induce rhythmic contractions in the actin-myosin fibers, as described in Section IV-B. Moreover, an edge $e_{i j} \in E$ denotes the virtual protoplasmic vein connecting two Physarum cells $v_{i}$ and $v_{j}$, and $l_{i j}=L\left(e_{i j}\right)$ denotes the length of $e_{i j}$.

Definition 9 (Flux Rule): Given a virtual protoplasmic vein $e_{i j}$ connecting two Physarum cells $v_{i}, v_{j} \in V$, the protoplasmic $f l u x^{7} q_{i j}^{\sigma}(t)$ induced by the stimulated Physarum cell $v_{\sigma} \in S$ through $e_{i j}$ at time $t$ is defined as follows:

$$
q_{i j}^{\sigma}(t)=\frac{d_{i j}(t)}{l_{i j}}\left(p_{i}^{\sigma}(t)-p_{j}^{\sigma}(t)\right)=-q_{j i}^{\sigma}(t)
$$

where $p_{i}^{\sigma}(t)$ is the protoplasmic pressure of vertex $v_{i}$ induced by $v_{\sigma} \in S$ at time $t, d_{i j}(t) \in \mathbb{R}_{0}^{+}$is the conductivity of link $e_{i j}$ at time $t$, and $l_{i j}=L\left(e_{i j}\right)$ is the length of vein $e_{i j}$.

Remark: The Flux Rule agrees with the Physarum physiology [8]: The flux of a protoplasmic soil can be approximated as a Poiseuille flow. Consequently, the protoplasmic flux can be expressed as $q_{i j}^{\sigma}(t)=\frac{\pi r_{i j}^{4}}{8 \eta} \frac{p_{i}^{\sigma}(t)-p_{j} \sigma(t)}{l_{i j}}$, where $l_{i j}$ and $r_{i j}$ are the length and the radius of the tubular structure, respectively, $\eta$ is the dynamic viscosity coefficient of the protoplasmic soil, and $p_{i}^{\sigma}(t)$ and $p_{j}^{\sigma}(t)$ are the pressures at time $t$ of the protoplasmic soil at the edges of the tube, induced by the rhythmic contractions of the Physarum cell $v_{\sigma} \in S$.

Definition 10 (Vertex Rule): Given a Physarum cell $v_{i}$, the algebraic sum of the protoplasmic fluxes $q_{i j}^{\sigma}(t)$ induced by the stimulated Physarum cell $v_{\sigma} \in S$ at time $t$ is

$$
\begin{aligned}
\sum_{j=1, j \neq i}^{n} q_{i j}^{\sigma}(t) & =\sum_{j=1, j \neq i}^{n} \frac{d_{i j}(t)}{l_{i j}}\left(p_{i}^{\sigma}(t)-p_{j}^{\sigma}(t)\right) \\
& = \begin{cases}f_{0}, & \text { if } v_{i} \in S \wedge i=\sigma \\
-\frac{f_{0}}{|S|-1}, & \text { if } v_{i} \in S \wedge i \neq \sigma \\
0, & \text { if } v_{i} \in V \backslash S\end{cases}
\end{aligned}
$$

where $\wedge$ denotes the logical operator and $V \backslash S$ denotes the complement of $S$ in $V, f_{0} \in \mathbb{R}^{+}$denotes the flux constant, and $|S|$ denotes the cardinality of set $S$.

Remark: The Vertex Rule agrees with the Physarum physiology. In fact, in a Physarum cell, the protoplasmic flow is

${ }^{7}$ If $q_{i j}^{\sigma}(t)>0$, the flux through $e_{i j}$ is oriented from $v_{i}$ to $v_{j}$ at time $t$. Otherwise, the flux is oriented from $v_{j}$ to $v_{i}$. 
caused by the hydrostatic pressure induced by the rhythmic contractions of actin-myosin fibers. When different nutrient sources are available, the actin-myosin fibers closer to the different nutrient sources contract with a different phase and/or amplitude, thus generating a protoplasmic flow through the organism.

Remark: According to the Vertex Rule, the cell $v_{\sigma} \in S$ induces the protoplasmic flow $f_{0}$, and the remaining $|S|-1$ cells in $S$ act as sinks for the flow. In fact, if $v_{i} \notin S$, then no flux is originated at $v_{i}$, i.e., the flux entering the vertex is equal to the flux leaving the vertex. Differently, if $v_{i} \in S$ and $i=\sigma$, then a constant positive flux $f_{0}$ is originated at $v_{i}$, whereas if $v_{i} \in S$ and $i \neq \sigma$, then $v_{i}$ acts as sink, and hence a negative flux is originated at $v_{i}$.

Definition 11 (Conductivity Rule): Given a virtual protoplasmic vein $e_{i j}$ connecting two Physarum cells $v_{i}, v_{j} \in V$, the conductivity value $d_{i j}(t)$ of $e_{i j}$ evolves over time according to the following rule8:

$$
\dot{d}_{i j}(t) \triangleq \frac{d}{d t} d_{i j}(t)=q_{i j}(t)-d_{i j}(t)
$$

with $q_{i j}(t)=\frac{1}{|S|} \sum_{v_{\sigma} \in S}\left|q_{i j}^{\sigma}(t)\right|$ denoting the arithmetic average of the absolute value of the protoplasmic flux $q_{i j}^{\sigma}(t)$ induced by $v_{\sigma} \in S$ at time $t, q_{i j}(t)$ denoting the average of the absolute values of the protoplasmic fluxes $q_{i j}^{\sigma}(t)$ flowing through link $e_{i j}$ at time $t$, and $|S|$ denoting the cardinality of set $S$.

Remark: The Conductivity Rule agrees with the Physarum polycephalum physiology [8]: Physarum tubular structures with larger fluxes become thicker, while those with smaller fluxes become thinner and eventually disappear when there is no protoplasmic flux through the tube. This effect is caused by the stretch activation induced by the protoplasmic flow over actin-myosin fibers. Consequently, we model the evolution of the conductivity as a function of the overall induced protoplasmic flux, i.e., as a function of the average absolute values of $q_{i j}^{\sigma}(t)$.

Remark: We note that, differently from the Physarum models available in literature such as [8], our model allows us to model the presence of rhythmic contractions in more than two Physarum cells: 1) by defining a protoplasmic flow for each contracting Physarum cell; 2) by combining the different protoplasmic flows at each link through the term $q_{i j}(t)$.

Definition 12 (Physarum BioNetwork for the Steiner Tree Problem): Given an undirected graph $G$, a length function $L: E \rightarrow \mathbb{R}^{+}$and a set $S$ of Steiner vertices, the Physarum BioNetwork for the Steiner Tree Problem is the system $\Upsilon(t)$

$$
\Upsilon(t)=(G, L, S, \mathbf{D}(0), t)
$$

which evolves according to (3)-(5), starting from the initial conditions $\mathbf{D}(0)=\left\{d_{i j}(0)\right\}_{e_{i j} \in E}$.

Remark: $\Upsilon(t)$ models a Physarum BioNetwork in which some Physarum cells, i.e., the cells hosted in the Steiner vertices, are stimulated with positive feedbacks to induce rhythmic contractions in the actin-myosin fibers [Fig. 5(a)].

Definition 13 (Physarum Steiner Tree Graph): Given a Physarum BioNetwork modeled by (6), the Physarum Steiner Tree Graph is the temporal undirected graph $G(t)=(V, E(t)) \subseteq G$, where $E(t)=\left\{e_{i j} \in E \mid q_{i j}(t) \neq 0\right\}$,

\footnotetext{
${ }^{8}$ We note that, for the sake of simplicity, we omit in (5) a dimensional factor for converting a flux quantity $q_{i j}(t)$ in a conductivity value $d_{i j}(t)$.
}

with $q_{i j}(t)$, defined in (5), denoting the average of the absolute values of the protoplasmic fluxes $q_{i j}^{\sigma}(t)$ flowing through link $e_{i j}$ at time $t$.

Remark: The Physarum Steiner Tree Graph $G(t)$ represents, at time $t$, the subset of the virtual protoplasmic veins of the Physarum BioNetwork characterized by a protoplasmic flux different from zero. In Section V, we prove that the Physarum Steiner Tree Graph $G(t)$ constituted by Physarum cycles evolves with an exponential convergence rate toward the minimum-length Steiner tree. In other words, we prove that a Physarum BioNetwork, in which the Steiner cells are stimulated to induce rhythmic contractions in the actin-myosin fibers, solves the Steiner tree problem.

\section{Physarum Bionetwork Network: Steiner Tree ANALYSIS}

In this section, we analytically prove that the Physarum Steiner Tree Graph $G(t)$ evolves toward the minimum-length Steiner tree with an exponential convergence rate. More in detail, we discuss in Section V-A the Physarum BioNetwork behavior in an arbitrary time instant. Then, in Section V-B, we discuss the Physarum BioNetwork Equilibrium and give the definition of Physarum cycle, whereas in Section V-C, we prove the main result (Theorem 2). In Section V-D we discuss the exponential convergence rate in terms of computation complexity. Finally, in Section V-E, we validate the proposed Physarum BioNetwork through a case study.

\section{A. Physarum BioNetwork Solutions}

Here, we prove with Theorem 1 that system (4) admits a unique solution if and only if the Physarum Steiner Tree Graph $G(t)$ spans all the vertices in $S$. Since the proof of Theorem 1 requires Lemmas $1-4$, we first give these preliminary results.

To this aim, let us consider the Physarum BioNetwork $\Upsilon(t)$ in an arbitrary time instant $t$. Moreover, let us suppose, without loss of generality, that $S=\left\{v_{1}, \ldots, v_{|S|-1}, v_{|S|}\right\}$, and let us adopt the following simplified notation: $G=G(t), p_{i}^{\sigma}=p_{i}^{\sigma}(t)$, $q_{i j}^{\sigma}=q_{i j}^{\sigma}(t)$, and $d_{i j}=d_{i j}(t)$ when $e_{i j} \in E, d_{i j}=0$ when $e_{i j} \notin E$.

Lemma 1: System (4) is an undetermined system.

Proof: See Appendix-A.

Since (4) is undetermined, for any Steiner vertex $v_{\sigma} \in S$ we can omit the $\sigma$ th row and rewrite (4) as

$$
\begin{aligned}
\sum_{j=1, j \neq i}^{n} q_{i j}^{\sigma} & =\sum_{j=1, j \neq i}^{n} \frac{d_{i j}}{l_{i j}}\left(p_{i}^{\sigma}-p_{j}^{\sigma}\right) \\
& = \begin{cases}-\frac{f_{0}}{|S|-1}, & \text { if } 1 \leq i<\sigma \\
0, & \text { if } \sigma<i \leq n .\end{cases}
\end{aligned}
$$

By setting $9 p_{\sigma}^{\sigma}=0$ for any $v_{\sigma} \in S$, and by denoting with $\mathbf{p}^{\sigma}=\left\{p_{1}^{\sigma}, \ldots, p_{\sigma-1}^{\sigma}, p_{\sigma+1}^{\sigma}, \ldots p_{n}^{\sigma}\right\}^{T}$ and $^{10}$ $\mathbf{f}^{\sigma}=\overbrace{\left\{-\frac{f_{0}}{|S|-1}, \ldots,-\frac{f_{0}}{|S|-1}\right.}^{|S|-1 \text { elements }}, \overbrace{0, \ldots, 0}^{n-|S| \text { elements }}\}^{T}$, we can

\footnotetext{
${ }^{9}$ This assumption is not restrictive since each flux depends on the difference between two pressure values.

${ }^{10}$ Although $\mathbf{f}^{\sigma}$ does not depend on $\sigma$, we adopt a $\sigma$-dependent notation for consistency.
} 
rewrite (7) as

$$
\underbrace{\left[\begin{array}{cccc}
\mathbf{A}^{1} & \mathbf{0} & \ldots & \mathbf{0} \\
\mathbf{0} & \mathbf{A}^{2} & \cdots & \mathbf{0} \\
\cdots & \cdots & \cdots & \cdots \\
\mathbf{0} & \mathbf{0} & \cdots & \mathbf{A}|\dot{S}|
\end{array}\right]}_{\mathbf{A} \in \mathcal{R}} \underbrace{\left[\begin{array}{c}
\mathbf{p}^{1} \\
\mathbf{p}^{2} \\
\cdots \\
\mathbf{p}^{|S|}
\end{array}\right]}_{\mathbf{p} \in \mathcal{R}^{|S|(n-1) \times 1} \times(n-1)}=\underbrace{\left[\begin{array}{c}
\mathbf{f}^{1} \\
\mathbf{f}^{2} \\
\cdots \\
\mathbf{f}^{|S|}
\end{array}\right]}_{\mathbf{f} \in \mathcal{R} S \mid(n-1) \times 1}
$$

where $\mathbf{A}^{\sigma}=\left\{a_{i j}\right\}_{i, j=1, i, j \neq \sigma}^{n}$ is a $n-1$ square matrix with $a_{i i}=\sum_{j=1, j \neq i}^{n} \frac{d_{i j}}{l_{i j}}$ and $a_{i j}=-\frac{d_{i j}}{l_{i j}}$ for $i \neq j$.

Lemma 2: For any $v_{\sigma} \in S$ and $v_{j} \in V$, it results $p_{j}^{\sigma} \leq 0$. Moreover, for any $v_{\sigma} \in S$ and $v_{i} \in S \backslash\left\{v_{\sigma}\right\}$, it results $p_{i}^{\sigma}<0$. Proof: See Appendix-B.

Lemma 3: For any $v_{\sigma} \in S$ and $v_{j} \in V \backslash S$, there exists at least a $v_{i} \in S \backslash\left\{v_{\sigma}\right\}$ such that $p_{i}^{\sigma}<p_{j}^{\sigma}$.

Proof: See Appendix-C.

Corollary 1: For any $v_{\sigma} \in S$, there exists at least a $v_{i} \in S$ $\backslash\left\{v_{\sigma}\right\}$ such that $p_{i}^{\sigma} \leq p_{j}^{\sigma}$ for any $v_{j} \in V$.

Proof: It follows directly from Lemma 3.

Lemma 4: For any $v_{\sigma} \in S$ and $v_{i}, v_{j} \in V$, we have $\left|q_{i j}^{\sigma}\right| \leq$ $f_{0}$.

Proof: See Appendix-D.

Theorem 1: System (7) admits a unique solution $\mathbf{p}=\left\{\mathbf{p}^{1}, \ldots, \mathbf{p}^{|S|}\right\}$ if and only if the vertices in $S$ are connected each other in the Physarum Steiner Tree Graph $G$.

Proof: See Appendix-E.

\section{B. Physarum BioNetwork Equilibrium}

Stemming from Theorem 1, in this section we discuss the equilibrium of the Physarum BioNetwork $\Upsilon(t)$. To this aim, we first restrict with Lemmas 5-7 the set of admissible values for the link conductivities $\mathbf{D}(t)=\left\{d_{i j}(t)\right\}_{v_{i}, v_{j} \in V}$ of the Physarum BioNetwork $\Upsilon(t)$

Lemma 5: If there exists $t_{0} \geq 0$ such that $e_{i j} \notin E\left(t_{0}\right)$, then $e_{i j} \notin E(t)$ for any $t \geq t_{0}$.

Proof: See Appendix-F.

Remark: Lemma 5 states that if there exists a time instant $t_{0}$ so that a link does not belong to the Physarum Steiner Tree Graph $G\left(t_{0}\right)$, then the link does not belong to $G(t)$ for any $t \geq t_{0}$.

Lemma 6: The hypercube $H$ = $\left\{\mathbf{D}(t)\left|0 \leq d_{i j}(t) \leq\right| S \mid f_{0} \forall e_{i j} \in E(t)\right\}$ is an attracting and invariant space for the link conductivities of the Physarum BioNetwork $\Upsilon(t)$ given in (6).

Proof: Since

$-d_{i j}(t) \leq \dot{d}_{i j}(t)=q_{i j}(t)-d_{i j}(t)=\sum_{v_{\sigma} \in S}\left|q_{i j}^{\sigma}(t)\right|-d_{i j}(t) \leq|S| f_{0}$

from (5) and Lemma 4, the thesis follows.

Remark: Lemma 6 states that for any initial conditions $\mathbf{D}(0) \in H$, for any $t \geq 0$, and for any $e_{i j} \in E$, we have $d_{i j}(t) \in H$.
Lemma 7: The lower bound of $\mathrm{H}\left\{\mathbf{D}(t) \mid d_{i j}(t)=0 \forall e_{i j} \notin E(t)\right\}$ is an invariant space for the link conductivities of the Physarum BioNetwork $\Upsilon(t)$ given in (6).

Proof: It follows directly from Lemma (5).

Remark: Stemming from Lemmas 6 and 7, in the following we assume without loss of generality that $\mathbf{D}(0)$ are feasible initial conditions, i.e., $\mathbf{D}(0)=\left\{d_{i j}(0)\left|0<d_{i j}(0) \leq\right| S \mid f_{0} \forall e_{i j} \in E\right\}$.

We now discuss the equilibrium of the Physarum BioNetwork $\Upsilon(t)$ starting from feasible initial conditions $\mathbf{D}(0)$, both when $G(t)$ is a tree (Lemma 8) and when $G(t)$ is not a tree (Lemma 9).

Remark: At the equilibrium, from (5) it follows that, when the conductivity $d_{i j}$ of an arbitrary link $e_{i j}$ is different from zero, the length $l_{i j}$ of such a link is equal to the average of the absolute values of the protoplasmic fluxes $q_{i j}^{\sigma}$

$$
\begin{gathered}
\dot{d}_{i j}=0 \Longleftrightarrow q_{i j}-d_{i j}=\frac{1}{|S|} \frac{d_{i j}}{l_{i j}} \sum_{v_{\sigma} \in S}\left|p_{i}^{\sigma}-p_{j}^{\sigma}\right|-d_{i j} \\
\Longleftrightarrow\left\{\begin{array}{l}
d_{i j}=q_{i j}>0 \wedge l_{i j}=\frac{1}{|S|} \sum_{v_{\sigma} \in S}\left|p_{i}^{\sigma}-p_{j}^{\sigma}\right| \\
d_{i j}=q_{i j}=0 .
\end{array}\right.
\end{gathered}
$$

Lemma 8: Let $\mathcal{T}=\left(V, E_{\mathcal{T}}\right) \subseteq G$ denote an arbitrary tree spanning all the vertices in $S$, and let $\mathbf{D}^{\mathcal{T}}=\left\{d_{i j}^{\mathcal{T}}\right\}_{e_{i j} \in E}$ be the link conductivities with

$$
\begin{cases}d_{i j}^{\mathcal{T}}>0 \wedge l_{i j}=\frac{1}{|S|} \sum_{v_{\sigma} \in S}\left|p_{i}^{\sigma}-p_{j}^{\sigma}\right|, & \text { if } e_{i j} \in E_{\mathcal{T}} \\ d_{i j}^{\mathcal{T}}=0, & \text { otherwise. }\end{cases}
$$

Then, we have the following

I) $\mathbf{D}^{\mathcal{T}}$ is an equilibrium point for the Physarum BioNetwork $\Upsilon(t)$.

II) For any $e_{i j} \in E_{\mathcal{T}}$, the protoplasmic flux $q_{i j}^{\sigma}$ induced by the Steiner vertex $v_{\sigma} \in S$ through $e_{i j}$ is given by

$$
q_{i j}^{\sigma}= \begin{cases}-f_{0} \frac{\left|S_{i j}^{\mathcal{T}}\right|}{|S|-1}=-f_{0} \frac{|S|-S_{j i}^{\mathcal{T}} \mid}{|S|-1}, & \text { if } q_{i j}^{\sigma}<0 \\ +f_{0} \frac{\left|S_{i j}^{\mathcal{T}}\right|}{|S|-1}, & \text { otherwise. }\end{cases}
$$

III) For any $e_{i j} \in E_{\mathcal{T}}$, the link conductivity $d_{i j}^{\mathcal{T}}$ is given by

$$
d_{i j}^{\mathcal{T}}=q_{i j}=\frac{f_{0}}{|S|} \frac{2\left|S_{i j}^{\mathcal{T}}\right|\left(|S|-\left|S_{i j}^{\mathcal{T}}\right|\right)}{|S|-1} .
$$

IV) For any $e_{i j} \in E_{\mathcal{T}}$, the protoplasmic pressure $p_{i}^{\sigma}$ at vertex $v_{i}$ induced by the Steiner vertex $v_{\sigma} \in S$ is given by

$$
p_{i}^{\sigma}= \begin{cases}p_{j}^{\sigma}-l_{i j} \frac{|S|}{2\left(|S|-\left|S_{i j}^{\mathcal{T}}\right|\right)}, & \text { if } q_{i j}^{\sigma}<0 \\ p_{j}^{\sigma}+l_{i j} \frac{|S|}{2\left(|S|-\left|S_{i j}^{\mathcal{T}}\right|\right)}, & \text { otherwise. }\end{cases}
$$

V) For any $v_{i}, v_{j} \in V$ so that $\exists P_{i j} \subset \mathcal{T}$, the average differential pressure between $v_{i}$ and $v_{j}$ is given by (14), shown at the bottom of the page,

$$
\frac{1}{|S|} \sum_{v_{\sigma} \in S}\left|p_{i}^{\sigma}-p_{j}^{\sigma}\right|=L\left(E\left(P_{i j}\right)\right)-\frac{1}{|S|} \cdot \sum_{v_{\sigma} \in S_{i k_{1}}^{\mathcal{T}} \bigcup S_{j k_{N-1}}^{\mathcal{T}}}\left(\left|p_{i}^{\sigma}-p_{k(\sigma)}^{\sigma}\right|+\left|p_{j}^{\sigma}-p_{k(\sigma)}^{\sigma}\right|-\left|p_{i}^{\sigma}-p_{j}^{\sigma}\right|\right)
$$


with

$$
\begin{aligned}
\left|p_{i}^{\sigma}-p_{k(\sigma)}^{\sigma}\right| & =\sum_{e_{k_{l} k_{l+1}} \in P_{i k(\sigma)}} l_{k_{l} k_{l+1}} \frac{|S|}{2\left(|S|-\left|S_{k_{l} k_{l+1}}^{\mathcal{T}}\right|\right)} \\
\left|p_{j}^{\sigma}-p_{k(\sigma)}^{\sigma}\right| & =\sum_{e_{k_{l} k_{l+1}} \in P_{j k(\sigma)}} l_{k_{l} k_{l+1}} \frac{|S|}{2\left(|S|-\left|S_{k_{l} k_{l+1}}^{\mathcal{T}}\right|\right)}
\end{aligned}
$$

and

$$
\begin{aligned}
\left|p_{i}^{\sigma}-p_{j}^{\sigma}\right|=\mid & \sum_{e_{k_{l} k_{l+1}} \in P_{i k(\sigma)}} l_{k_{l} k_{l+1}} \frac{|S|}{2\left(|S|-\left|S_{k_{l} k_{l+1}}^{\mathcal{T}}\right|\right)}- \\
& -\sum_{e_{k_{l} k_{l+1}} \in P_{j k(\sigma)}} l_{k_{l} k_{l+1}} \frac{|S|}{2\left(|S|-\left|S_{k_{l} k_{l+1}}^{\mathcal{T}}\right|\right)} \mid
\end{aligned}
$$

where $S_{i j}^{\mathcal{T}} \subset S$ denotes the set ${ }^{11}$ of Steiner vertices in $S$ connected with $v_{i} \in V$ in the subgraph $\left(V, E_{\mathcal{T}} \backslash\left\{e_{i j}\right\}\right)$, $P_{i j}$ is the simple path connecting $v_{i}$ and $v_{j}$ with $E_{P_{i j}}=$ $\left\{e_{k_{l} k_{l+1}}\right\}_{l=0}^{N-1}$, and $v_{k(\sigma)} \in P_{i j}$ is the vertex closest to $v_{\sigma}$.

Proof: See Appendix-G.

Remark: We note that, if $\exists P_{i j}^{d} \subseteq \mathcal{T}$, i.e., if $v_{i}$ and $v_{j}$ are connected in $\mathcal{T}$ with a direct path, then (14) reduces to

$$
\frac{1}{|S|} \sum_{v_{\sigma} \in S}\left|p_{i}^{\sigma}-p_{j}^{\sigma}\right|=L\left(E\left(P_{i j}\right)\right) .
$$

Definition 14 (Physarum Cycle): Given a Physarum Steiner Tree Graph $G(t)$ and the corresponding minimum-length Steiner tree $\mathcal{T}_{\text {opt }}=\left(V, E_{\mathcal{T}_{\text {opt }}}\right)$, a cycle $\mathcal{C}=\left(V, E_{\mathcal{C}}\right) \subseteq G(t)$ between two Steiner vertices $v_{i}, v_{j} \in S$ is a Physarum cycle if we have the following.

Case 1) $\left|S_{\mathcal{C}}\right|=0$, it results

$$
L\left(E_{P_{i j}^{d, 2}}\right)>L\left(E_{P_{i j}^{d, 1}}\right)
$$

where $S_{\mathcal{C}}$ denotes the set of Steiner vertices in $S$ $\backslash\left\{v_{i}, v_{j}\right\}$ belonging to $\mathcal{C}$, and $P_{i j}^{d, 1}=\left(V, E_{P_{i j}^{d, 1}}\right)$ and $P_{i j}^{d, 2}=\left(V, E_{P_{i j}^{d, 2}}\right)$ denote the two direct paths ${ }^{12}$ between $v_{i}$ and $v_{j}$ in $\mathcal{C}$, respectively.

Case 2) $\left|S_{\mathcal{C}}\right|>0: \exists v_{k}, v_{l} \in S_{\mathcal{C}} \bigcup\left\{v_{i}, v_{j}\right\}$

$$
\begin{aligned}
& L\left(E_{P_{k l}^{d}}\right)>L\left(E_{P_{m s}^{d}}\right) \quad \forall v_{m}, v_{s} \in S_{\mathcal{C}} \bigcup\left\{v_{i}, v_{j}\right\} \\
& L\left(E_{P_{k l}^{d}}\right)>L\left(E_{P_{k l}^{i}}\right)-\frac{1}{|S|} \sum_{\substack{v_{\sigma} \notin S_{k P_{k l}^{d}}^{\tau_{\mathrm{opt}}} \bigcup S_{l P_{l k}^{d}}^{\tau_{\mathrm{opt}}}\\
}}\left(\left|p_{k}^{\sigma}-p_{k(\sigma)}^{\sigma}\right|\right. \\
& \left.+\left|p_{l}^{\sigma}-p_{k(\sigma)}^{\sigma}\right|-\left|p_{k}^{\sigma}-p_{l}^{\sigma}\right|\right)
\end{aligned}
$$

where $S_{\mathcal{C}}$ denotes the set of Steiner vertices in $S \backslash$ $\left\{v_{i}, v_{j}\right\}$ belonging to $\mathcal{C}, P_{k l}^{d}=\left(V, E_{P_{k l}^{d}}\right)$ and $P_{k l}^{i}=$ $\left(V, E_{P_{k l}^{i}}\right)$ denote the direct and the indirect paths ${ }^{13}$

\footnotetext{
${ }^{11}$ If $v_{i} \in S$, then $v_{i} \in S_{i j}^{\mathcal{T}}$.

${ }^{12}$ If more than two direct paths exist, i.e., the Physarum cycle is not a simple cycle, then it must exist a unique shortest direct path.

${ }^{13}$ If more than two indirect paths exist, i.e., the Physarum cycle is not a simple cycle, then (18) must hold for any indirect path.
}

between $v_{k}$ and $v_{l}$ in $\mathcal{C}$, respectively, $S_{k P_{k l}^{d}}^{\mathcal{T}_{\mathrm{opt}}}$ denotes the set ${ }^{14}$ of Steiner vertices in $S$ connected with $v_{k}$ in the subgraph $\left(V, E_{\mathcal{T}_{\text {opt }}} \backslash E_{P_{k l}^{d}}\right)$, and, finally, $v_{k(\sigma)} \in$ $P_{k l}^{i}$ is the closest vertex to $v_{\sigma}$.

Remark: The first condition (16) assures the existence of a unique shortest direct path between a pair of Steiner vertices. This condition is necessary since, if multiple shortest direct paths exist, then the number of equilibrium points for the Physarum BioNetwork $\Upsilon(t)$ could be uncountably infinite. In fact, denoted with $q_{P_{i j}^{d}}$ the protoplasmic flux between the Steiner vertices $v_{i}$ and $v_{j}$ when only one shortest direct path is available, then the set of equilibrium points is as follows:

$$
\begin{aligned}
& q_{P_{i j}^{d, 1}}=\alpha q_{P_{i j}^{d}} \\
& q_{P_{i j}^{d, 1}}=(1-\alpha) q_{P_{i j}^{d}}
\end{aligned} \quad \alpha \in[0,1] .
$$

The second condition (17), (18) assures the existence of a pair of Steiner vertices whose direct path is longer than the average differential pressure between those vertices in the minimumlength Steiner tree $\mathcal{T}_{\text {opt }}$. This condition is needed by Lemma 9 to assure that the interior of $H$ does not contain any equilibrium point for the Physarum BioNetwork $\Upsilon(t)$.

Lemma 9: Let $G(t)$ denote a graph spanning all the vertices in $S$ where any cycle is a Physarum cycle. Then, if $G(t)$ is not a tree, the interior of $H$ does not contain any equilibrium point for the Physarum BioNetwork $\Upsilon(t)$.

Proof: See Appendix-H.

\section{Physarum BioNetwork Convergence}

Finally, we can prove in Theorem 2 that the Physarum Steiner Tree Graph $G(t)$ constituted by Physarum cycles evolves toward the minimum-length Steiner tree with an exponential convergence rate. Since the proof of Theorem 2 requires Lemma 10, we first give this preliminary result.

Lemma 10: Let $G(t)$ denote a graph spanning all the vertices in $S$ where any cycle is a Physarum Cycle. If $G(t)$ is not a tree, there exists at least an edge $e_{i j} \in E(t) \backslash E_{\mathcal{T}_{\mathrm{opt}}}$ so that $d_{i j}(t)$ exponentially converges to zero.

Proof: See Appendix-I.

Remark: Lemma 10 proves that any link not belonging to the minimum-length Steiner tree disappears from $G(t)$, and the rate of such a convergence is exponential.

Theorem 2: Let $G(t)$ denote a graph spanning all the vertices in $S$ where any cycle is a Physarum Cycle. Then, the equilibrium point $\mathbf{D}_{\mathrm{opt}}^{\mathcal{T}}$ associated with the minimum-length Steiner tree $\mathcal{T}_{\text {opt }}$ is globally asymptotically stable for the Physarum BioNetwork $\Upsilon(t)$.

Proof: It follows by iteratively applying Lemmas 9 and 10 to $G(t)$.

Remark: Clearly, the exponential convergence rate is a very attractive feature of the Physarum BioNetwork $\Upsilon(t)$ since it allows $\Upsilon(t)$ to efficiently compute the minimum-length Steiner tree, as discussed in Section V-D.

Remark: We argue that there exists a relationship between the Physarum cycle condition and the choice of the artificially stimulated Physarum cells. However, further research is necessary to establish if this correlation exists.

$$
{ }^{14} \text { If } v_{k} \in S \text {, then } v_{k} \in S_{k P_{k l}^{d}}^{\mathcal{T}_{\mathrm{opt}}} .
$$




\section{Computational Complexity}

In the following, we first discuss the computational power of the Physarum BioNetwork. Then, we discuss the worst-case complexity of the Physarum BioNetwork in solving the Steiner tree problem.

Definition 15 (BioNetwork Computation Model): A computing device is defined as a device mapping an input to an output [15]. Consequently, the BioNetwork can be considered a computing device mapping the input, i.e., the initial condition $\Upsilon(0)=(G, L, S, \mathbf{D}(0), 0)$ defined in (6), to the output, i.e., the equilibrium point $\mathbf{D}_{\text {opt }}^{\mathcal{T}}$.

Definition 16 (BioNetwork Complete Computation): The BioNetwork computation is complete when an $\epsilon_{p}$-vicinity of the equilibrium point is approached and the BioNetwork is confined there.

Remark: The above definition allows a fair comparison to traditional computation theory [15]. Specifically, it allows a fair comparison to a traditional computer architecture with $p$ bits of precision and a machine epsilon equal to $\epsilon_{p}$.

Proposition 1 (BioNetwork Computation Power): Let $G(t)$ denote a graph spanning all the vertices in $S$ where any cycle is a Physarum Cycle. If $G(t)$ is not a tree, then there exists at least an edge $e_{i j} \in E(t) \backslash E_{\mathcal{T}_{\text {opt }}}$ so that the Physarum BioNetwork $\Upsilon(t)$ computes $d_{i j}$ in $p \tau_{i j} \log 2$ time units.

Proof: From (47) in Appendix-I, the rate of convergence toward the attractor $d_{i j}=0$ for $e_{i j} \in E(t) \backslash E_{\mathcal{T}_{\text {opt }}}$ satisfies the following condition:

$$
\left|d_{i j}(t)-d_{i j}\right|<e^{-\frac{1}{\tau_{i j}} t}
$$

where the exponential factor $\frac{1}{\tau_{i j}}$ is function of $L\left(E_{P}\right)-$ $L\left(E_{P_{\text {opt }}}\right)$, i.e., it is function of the difference between the length of the path $E_{P} \in G(t) \backslash \mathcal{T}_{\text {opt }}$ containing $e_{i j}$ and the length of the corresponding path $E_{P_{\mathrm{opt}}} \in \mathcal{T}_{\text {opt }}$ (see Appendix-I for further details). Thus, every $\tau_{i j} \log 2$ time units, a new bit of the attractor is computed [15], [16]. Consequently, according to Definition 16, the computation of $d_{i j}$ is completed in $p \tau_{i j} \log 2$ time units.

Proposition 2 (BioNetwork Time Complexity): Let $G(t)$ denote a graph spanning all the vertices in $S$ where any cycle is a Physarum Cycle, let $|E|$ denote the cardinality of set $E$, and let us refer to $\tau=\sup _{e_{i j} \in E} \tau_{i j}$ as the characteristic time of the problem. The Physarum BioNetwork $\Upsilon(t)$ computes the minimum-length Steiner tree $\mathcal{T}_{\text {opt }}$ with a worst-case time complexity upper-bounded by $|E| p \tau \log 2$.

Proof: The proof follows directly from Proposition 1 by accounting for Definition 16.

Remark: Further work is required to analyze the impact of the Physarum cycle condition on the proposed solution. Specifically, further research is needed to analyze the convergence and the complexity of the proposed solution in graphs where not all the cycles are Physarum cycles.

\section{E. Case Study}

Here, we validate the proposed Physarum BioNetwork through simulations. More specifically, we simulate a discrete-time BioNetwork constituted by Physarum cells, as described in Section IV. The cells are connected to each other

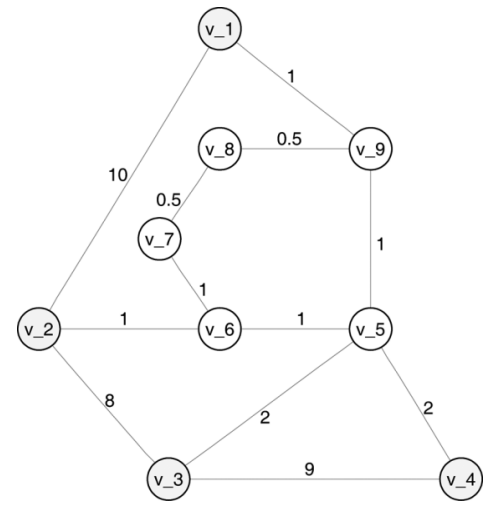

Fig. 6. Physarum Steiner Tree Graph $G(t)$ at time $t=0$. The gray circles denote the Steiner vertices, and the edge labels denote the edge lengths.

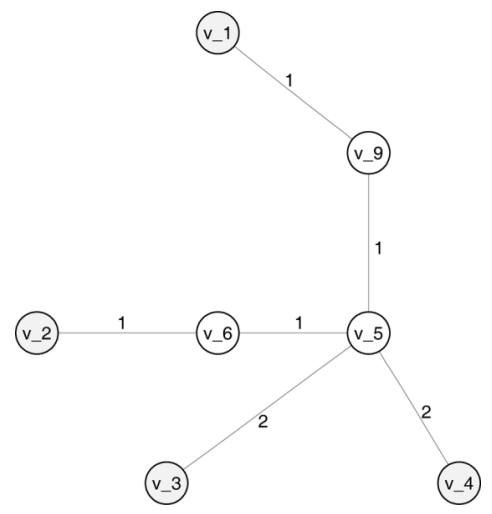

Fig. 7. Physarum Steiner Tree Graph $G(t)$ at time $t=20$.

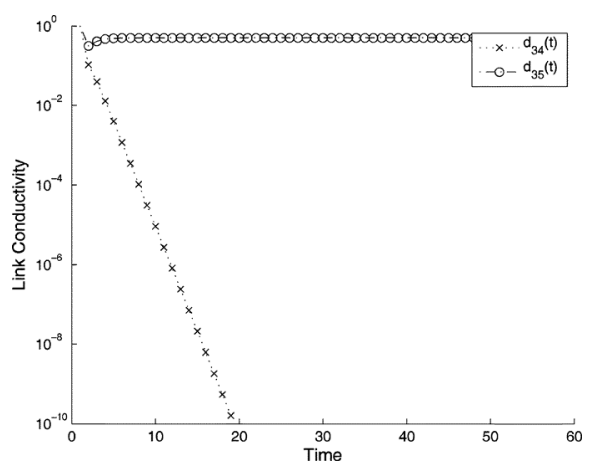

Fig. 8. Link conductivity as function of time for the topology in Fig. 6.

through bio-interfaces and network links, ${ }^{15}$ and the protoplasmic flow generated at each cell is virtually propagated along the entire network according to the network topology.

In the first experiment, we consider a network topology (Fig. 6) used in a widely cited work [17]. We simulate the evolution of the Physarum BioNetwork $\Upsilon(t)$ as a function of the discrete time. As shown in Fig. 7, the Physarum Steiner Tree Graph $G(t)$ evolves toward the minimum-length Steiner tree. Fig. 8 presents the link conductivity values as a function of the

\footnotetext{
${ }^{15}$ We assume that the delays generated by the system cascade bio-interface-network link-bio-interface are negligible compared to the actin-myosin fiber contraction time periods. This assumption is not restrictive since researchers have estimated the duration of actin-myosin fiber contraction periods in $2 \mathrm{~min}$.
} 


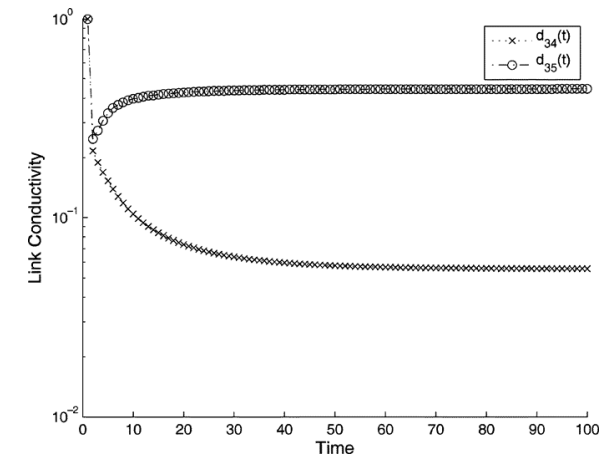

Fig. 9. Link conductivity as function of time for the topology in Fig. 6 when $l_{34}=2.5$.

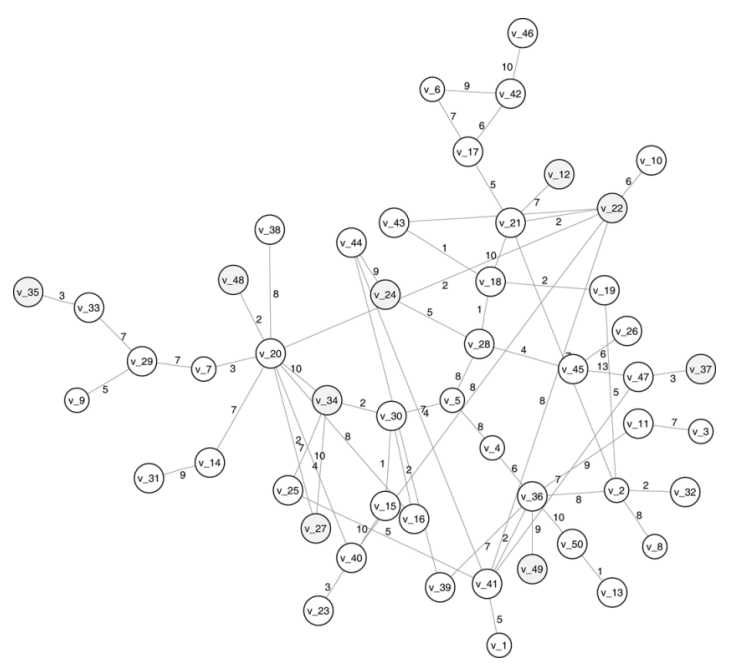

Fig. 10. Physarum Steiner Tree Graph $G(t)$ at time $t=0$. The gray circles denote the Steiner vertices, and the edge labels denote the edge lengths.

discrete time, with a logarithmic scale on the $y$-axis. More in detail, we consider two links: 1) the link $e_{35}$, belonging to the minimum-length Steiner tree $\mathcal{T}_{\text {opt }} ; 2$ ) the link $e_{34}$, not belonging to the minimum-length Steiner tree. First, we observe that the numerical results agree with Theorem 2: The conductivities of the links not belonging to the minimum-length Steiner tree go exponentially to zero as time increases. Moreover, we observe that Proposition 1 is confirmed by the numerical results: Every constant time period, a new bit of the attractor $d_{34}=0$ is computed.

In the second experiment, we still consider the network topology shown in Fig. 6, but we change the length of the link $e_{34}$ so that the cycle between the nodes $v_{3}, v_{4}$, and $v_{5}$ is not a Physarum cycle. More in detail, we set $l_{34}$ equal to the right side of (17), i.e., $l_{34}=2.5$. Clearly, since the hypotheses of Lemma 9 are not satisfied, there exists an equilibrium point when $G(t)$ is not a tree. Fig. 9 presents the values of the link conductivity as a function of the discrete time, with a logarithmic scale on the $y$-axis, for the links $e_{34}$ and $e_{35}$. First, we observe that for low values of time, the numerical results agree with Theorem 2: The conductivities of the links evolve with an exponential rate. However, since there exists a non-Physarum cycle, then there exists an equilibrium point when $G(t)$ is not a tree. More specifically, when $d_{34}(t)=1 / 1.8$ and $d_{35}=1 / 2.25$, the Physarum BioNetwork $\Upsilon(t)$ is at the

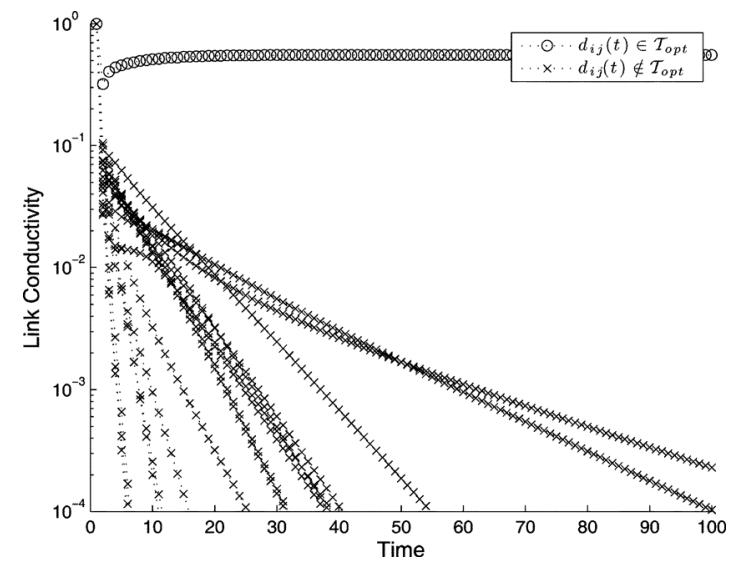

Fig. 11. Link conductivity as function of time for the topology in Fig. 10.

equilibrium. Finally, in the third experiment, we consider a larger network topology (Fig. 10) used in a previous work [18] (b01 topology). The Physarum Steiner Tree Graph $G(t)$ evolves toward the minimum-length Steiner tree with length equal to 82. Fig. 11 presents the link conductivity values as a function of the discrete time, with a logarithmic scale on the $y$-axis. More in detail, we consider two classes of links: 1) the class of links belonging to the minimum-length Steiner tree $\mathcal{T}_{\text {opt }}$, represented by link $\left.e_{12,21} ; 2\right)$ the links not belonging to the minimum-length Steiner tree. Similarly to the first experiment, the numerical results agree with Theorem 2 since the conductivities of the links not belonging to $\mathcal{T}_{\text {opt }}$ go exponentially to zero as time increases. Moreover, we observe that the numerical results agree with Propositions 1 and 2. Specifically, for any $e_{i j} \notin \mathcal{T}_{\text {opt }}$ : 1) every constant time period a new bit of the attractor $d_{i j}=0$ is computed; 2) the time period necessary for computing a new bit of the attractor depends on $e_{i j}$.

\section{CONCLUSION}

In this paper, the BioNetwork paradigm, a novel communication network paradigm in which the traditional network nodes are replaced by biological organisms, has been proposed. The BioNetwork paradigm aims at exploiting the evolutionary cleverness of nature through a community of living organisms that interact with each other. The BioNetwork paradigm has a huge potential since it can be adopted in many different applications. In the paper, this potential has been disclosed by proving that a BioNetwork can solve one of the most fundamental problems in networks, i.e., the Steiner tree problem. To this aim, a BioNetwork constituted by a unicellular organism, the Physarum polycephalum slime mold, has been designed, and it has been analytically proved that a Physarum BioNetwork can solve the Steiner tree problem in graphs constituted by Physarum cycles with an exponential convergence rate toward the optimal solution. The theoretical analysis has been validated through simulations.

\section{APPENDIX}

\section{A. Proof of Lemma 1}

We prove the lemma by showing that, for an arbitrary $v_{\sigma} \in S$, the $\sigma$ th row of system (4) is a linear combination of the other 
$n-1$ rows, i.e.,

$\sum_{j=1, j \neq \sigma}^{n} q_{\sigma j}^{\sigma}+\sum_{i=1, i \neq \sigma}^{n} \sum_{j=1, j \neq i}^{n} q_{i j}^{\sigma}=f_{0}-(|S|-1) \frac{f_{0}}{|S|-1}=0$.

Since $q_{i j}^{\sigma}=-q_{j i}^{\sigma}$ from (3), we can write the left side of (21) as in (22), shown at the bottom of the page.

\section{B. Proof of Lemma 2}

Thesis $1\left(p_{j}^{\sigma} \leq 0 \forall v_{\sigma} \in S, v_{j} \in V\right)$ : Since $p_{\sigma}^{\sigma}=0 \forall v_{\sigma} \in$ $S$, we prove the thesis with a reductio ad absurdum by supposing that $\exists v_{\sigma} \in S, v_{j} \in V \backslash\left\{v_{\sigma}\right\}: p_{j}^{\sigma}>0$. Without loss of generality, let us suppose that $p_{j}^{\sigma} \geq p_{k}^{\sigma} \forall v_{k} \in V$. It follows $\sum_{k=1, k \neq j}^{n} q_{j k}^{\sigma}>0$, and since $\sum_{k=1, k \neq j}^{n} q_{j k}^{\sigma} \leq 0 \forall v_{\sigma} \in$ $S, v_{j} \in V \backslash\left\{v_{\sigma}\right\}$ from (7), we obtain a reductio ad absurdum.

Thesis $2\left(p_{i}^{\sigma}<0 \forall v_{\sigma} \in S, v_{i} \in S \backslash\left\{v_{\sigma}\right\}\right)$ : Since $p_{k}^{\sigma} \leq$ $0 \forall v_{k} \in V$ from Thesis 1 , we prove the thesis with a reductio ad absurdum by supposing that $\exists v_{\sigma} \in S, v_{i} \in S \backslash\left\{v_{\sigma}\right\}: p_{i}^{\sigma}=0$. Again from Thesis 1, it follows $\sum_{k=1, k \neq i}^{n} q_{i k}^{\sigma} \geq 0$, and since $\sum_{k=1, k \neq i}^{n} q_{i k}^{\sigma}<0 \forall v_{i} \in S \backslash\left\{v_{\sigma}\right\}$ from (7), we obtain a reductio ad absurdum.

\section{Proof of Lemma 3}

We prove the lemma with a reductio ad absurdum by supposing that $\exists v_{\sigma} \in S, v_{j} \in V \backslash S: p_{j}^{\sigma} \leq p_{i}^{\sigma} \forall v_{i} \in S \backslash\left\{v_{\sigma}\right\}$. Without loss of generality, let us assume that $p_{j}^{\sigma} \leq p_{k}^{\sigma} \forall v_{k} \in V$. Thus, it follows

$$
\sum_{k=1, k \neq j}^{n} q_{j k}^{\sigma}=\sum_{k=1, k \neq j}^{n} \frac{d_{j k}}{l_{j k}}\left(p_{j}^{\sigma}-p_{k}^{\sigma}\right) \leq \frac{d_{j \sigma}}{l_{j \sigma}}\left(p_{j}^{\sigma}-p_{\sigma}^{\sigma}\right)<0 .
$$

Since $\sum_{k=1, k \neq j}^{n} q_{j k}^{\sigma}=0 \forall v_{j} \in V \backslash S$ from (7), we obtain a reductio ad absurdum.

\section{Proof of Lemma 4}

We prove the lemma by considering an arbitrary $v_{\sigma} \in S$, and by introducing a sequence of $n$ distinct indices $\left\{k_{i}^{\sigma}: v_{k_{i}^{\sigma}} \in V\right\}_{i=1}^{n}$ such that $k_{n}^{\sigma}=\sigma$ and $p_{k_{i}^{\sigma}}^{\sigma} \leq p_{k_{i+1}^{\sigma}}^{\sigma}$ for any $v_{i} \in V$.

We first consider the index $k_{n}^{\sigma}=\sigma$ and, according to (4), we have that $\sum_{j=1, j \neq k_{n}^{\sigma}}^{n} q_{k_{n}^{\sigma} k_{j}^{\sigma}}^{\sigma}=f_{0}$, and since $p_{k_{n}^{\sigma}}^{\sigma} \geq p_{k_{j}^{\sigma}}^{\sigma}$ for any $v_{j} \in V \backslash\left\{v_{\sigma}\right\}$ implies $q_{k_{n}^{\sigma} k_{j}^{\sigma}}^{\sigma} \geq 0$ for any $v_{j} \in V \backslash\left\{v_{\sigma}\right\}$, we have $\left|q_{\sigma j}^{\sigma}\right| \leq f_{0}$ for any $v_{j} \in V$.

Let us now consider the index $k_{i}^{\sigma}, i \neq n$, and let $\xi_{k_{i}^{\sigma}}^{\sigma}$ denote the flux indicator variable, i.e., $\xi_{k_{i}^{\sigma}}^{\sigma}=-\frac{f_{0}}{|S|-1}$ if $v_{k_{i}^{\sigma}} \in S \backslash\left\{v_{\sigma}\right\}$, $\xi_{k_{i}^{\sigma}}^{\sigma}=0$ otherwise.

According to (4), we obtain (23), shown at the bottom of the page.

Since we have

$$
q_{k_{i-2}^{\sigma} k_{i}^{\sigma}}^{\sigma}+q_{k_{i-2}^{\sigma} k_{i-1}^{\sigma}}^{\sigma} \geq \sum_{j=i-1}^{n} q_{k_{i-2}^{\sigma} k_{j}^{\sigma}}^{\sigma}=\sum_{j=1}^{i-3} q_{k_{j}^{\sigma} k_{i-2}^{\sigma}}^{\sigma}+\xi_{k_{i-2}^{\sigma}}^{\sigma}
$$

by substituting (24) in (23), it follows:

$$
\sum_{j=i+1}^{n} q_{k_{i}^{\sigma} k_{j}^{\sigma}}^{\sigma}=\sum_{j=1}^{i-3}\left(q_{k_{j}^{\sigma} k_{i}^{\sigma}}^{\sigma}+q_{k_{j}^{\sigma} k_{i-1}^{\sigma}}^{\sigma}+q_{k_{j}^{\sigma} k_{i-2}^{\sigma}}^{\sigma}\right)+\sum_{j=i-2}^{i} \xi_{k_{j}^{\sigma}}^{\sigma} .
$$

Iteratively, we obtain

$$
\begin{aligned}
\sum_{j=i+1}^{n} q_{k_{i}^{\sigma} k_{j}^{\sigma}}^{\sigma} & \geq \ldots \geq \sum_{j=1}^{i} q_{k_{1}^{\sigma} k_{j}^{\sigma}}^{\sigma}+\sum_{j=2}^{i} \xi_{k_{j}^{\sigma}}^{\sigma} \\
& \geq \sum_{j=1}^{n} q_{k_{1}^{\sigma} k_{j}^{\sigma}}^{\sigma}+\sum_{j=2}^{i} \xi_{k_{i}^{\sigma}}^{\sigma}=\sum_{j=1}^{i} \xi_{k_{i}^{\sigma}}^{\sigma} \geq-f_{0}
\end{aligned}
$$

$$
\begin{aligned}
\sum_{j=1, j \neq \sigma}^{n} q_{\sigma j}^{\sigma}+\sum_{i=1, i \neq \sigma}^{n} \sum_{j=1, j \neq i}^{n} q_{i j}^{\sigma} & =\sum_{j=1, j \neq \sigma}^{n} q_{\sigma j}^{\sigma}+\sum_{i=1, i \neq \sigma}^{n} q_{i \sigma}^{\sigma}+\sum_{i=1, i \neq \sigma}^{n} \sum_{j=1, j \neq i, \sigma}^{n} q_{i j}^{\sigma}=\sum_{i=1, i \neq \sigma}^{n} \sum_{j=1, j \neq i, \sigma}^{n} q_{i j}^{\sigma} \\
& =\sum_{j=1, j \neq \sigma}^{n-1} q_{n j}^{\sigma}+\sum_{i=1, i \neq \sigma}^{n-1} \sum_{j=1, j \neq i, \sigma}^{n} q_{i j}^{\sigma} \sum_{j=1, j \neq \sigma}^{n-1} q_{n j}^{\sigma}+\sum_{i=1, i \neq \sigma}^{n-1} q_{i n}^{\sigma}+\sum_{i=1, i \neq \sigma \quad j=1, j \neq i, \sigma}^{n-1} \sum_{i j}^{n-1} q_{j=1, i \neq \sigma}^{\sigma} \sum_{j=1, j \neq i, \sigma}^{2} q_{i j}^{\sigma}=0 . \\
& =\sum_{i=1, i \neq \sigma}^{n-1} \sum_{j=1, j \neq i, \sigma}^{n-1} q_{i j}^{\sigma}=\cdots=\sum_{i=1,1}^{2}
\end{aligned}
$$

$$
\begin{aligned}
\sum_{j=i+1}^{n} q_{k_{i}^{\sigma} k_{j}^{\sigma}}^{\sigma} & =\sum_{j=1}^{i-1} q_{k_{j}^{\sigma} k_{i}^{\sigma}}^{\sigma}+\xi_{k_{i}^{\sigma}}^{\sigma}=\sum_{j=1}^{i-2} q_{k_{j}^{\sigma} k_{i}^{\sigma}}^{\sigma}+q_{k_{i-1}^{\sigma} k_{i}^{\sigma}}^{\sigma}+\xi_{k_{i}^{\sigma}}^{\sigma} \geq \sum_{j=1}^{i-2} q_{k_{j}^{\sigma} k_{i}^{\sigma}}^{\sigma}+\sum_{j=i}^{n} q_{k_{i-1}^{\sigma} k_{j}^{\sigma}}^{\sigma}+\xi_{k_{i}^{\sigma}}^{\sigma} \\
& =\sum_{j=1}^{i-2} q_{k_{j}^{\sigma} k_{i}^{\sigma}}^{\sigma}+\sum_{j=1}^{i-2} q_{k_{j}^{\sigma} k_{i-1}^{\sigma}}^{\sigma}+\xi_{k_{i-1}^{\sigma}}^{\sigma}+\xi_{k_{i}^{\sigma}}^{\sigma}=\sum_{j=1}^{i-3}\left(q_{k_{j}^{\sigma} k_{i}^{\sigma}}^{\sigma}+q_{k_{j}^{\sigma} k_{i-1}^{\sigma}}^{\sigma}\right)+\left(q_{k_{i-2}^{\sigma} k_{i}^{\sigma}}^{\sigma}+q_{k_{i-2}^{\sigma} k_{i-1}^{\sigma}}^{\sigma}\right)+\sum_{j=i-1}^{i} \xi_{k_{j}^{\sigma}}^{\sigma}
\end{aligned}
$$


which implies $q_{k_{i}^{\sigma} k_{j}^{\sigma}}^{\sigma} \geq-f_{0} \forall i \neq n, j \neq i: k_{i}^{\sigma}<k_{j}^{\sigma}$. By exploiting the properties $q_{k_{i}^{\sigma} k_{j}^{\sigma}}^{\sigma} \leq 0$ and $q_{k_{i}^{\sigma} k_{j}^{\sigma}}^{\sigma}=-q_{k_{i}^{\sigma} k_{j}^{\sigma}}^{\sigma}$, the thesis follows.

\section{E. Proof of Theorem 1}

Case $1(\Rightarrow)$ : We prove the sufficient condition with a reductio ad absurdum by supposing that $\exists v_{\sigma} \in S, v_{i} \in S \backslash\left\{v_{\sigma}\right\}$ such that $v_{i}$ is not connected with $v_{\sigma}$ in $G$.

Let us denote with $\mathcal{V}_{i} \subset V \backslash\left\{v_{\sigma}\right\}$ the set of vertices connected with $v_{i}$ in $G$, and with $v_{m} \in \mathcal{V}_{i}: p_{m}^{\sigma}=\min _{v_{j} \in \mathcal{V}_{i}}\left\{p_{j}^{\sigma}\right\}$. Since $q_{m k}^{\sigma}=0 \forall v_{k} \in V: p_{k}^{\sigma}<p_{m}^{\sigma}$, it follows:

$$
\sum_{k=1, k \neq m}^{n} q_{m k}^{\sigma}=\sum_{k=1, k \neq m}^{n} \frac{d_{m k}}{l_{m k}}\left(p_{m}^{\sigma}-p_{k}^{\sigma}\right) \leq 0
$$

and, since $\sum_{j=1, j \neq m}^{n} q_{m j}^{\sigma} \geq 0 \forall v_{m} \in V \backslash\left\{v_{\sigma}\right\}$ from (7), it follows $q_{m k}^{\sigma}=0 \forall v_{k} \in V$. Thus, we have a reductio ad absurdum since $\exists v_{j} \in \mathcal{V}_{i}: q_{j m}^{\sigma}>0$ for reductio's hypothesis.

Case $2(\Leftarrow)$ : System $(8)$ admits a unique solution for any $\mathbf{f}$ if and only if $\mathbf{A}$ is nonsingular [19]. Since $\mathbf{A}$ is nonsingular if it is positive definite [19], we prove the necessary condition with a reductio ad absurdum by supposing that $S$ is connected in the Physarum Steiner Tree Graph $G$ and $\mathbf{A}$ is not positive definite, i.e., a $\mathbf{p} \neq \mathbf{0}$ such that $\mathbf{p}^{T} \mathbf{A} \mathbf{p}=\sum_{\sigma=1}^{|S|} \mathbf{p}^{\sigma T} \mathbf{A}^{\sigma} \mathbf{p}^{\sigma} \leq$ 0 exists. Clearly, this implies that there exists at least a $v_{\sigma} \in S$ : $\mathbf{p}^{\sigma T} \mathbf{A}^{\sigma} \mathbf{p}^{\sigma} \leq 0$ and, since $p_{\sigma}^{\sigma}=0$ and $d_{j k}=d_{k j}$, (28), shown at the bottom of the page, follows.

Let us denote with $v_{M} \in S: p_{M}^{\sigma}=\max _{v_{i} \in S}\left\{p_{i}^{\sigma}\right\}$ and with $\mathcal{V}_{M} \subseteq V$ the set of vertices connected with $v_{M}$. Since $p_{M}^{\sigma}>0$ from Lemma 2, we have that $\mathbf{p}^{\sigma T} \mathbf{A}^{\sigma} \mathbf{p}^{\sigma}=0$ implies

$$
\forall v_{k} \in V \backslash\left\{v_{M}\right\}: d_{M k}=0 \vee p_{M}^{\sigma}=p_{k}^{\sigma} \Longleftrightarrow \mathcal{V}_{M}=\emptyset
$$

where $\vee$ denotes the logical operator or. Thus, we have a reductio ad absurdum since $S \subseteq \mathcal{V}_{M}$ for reductio's hypothesis.

\section{F. Proof of Lemma 5}

Since from (5) we have that $e_{i j} \notin E\left(t_{0}\right)$ implies

$$
q_{i j}\left(t_{0}\right)=0 \Longrightarrow \dot{d}_{i j}\left(t_{0}\right)=q_{i j}\left(t_{0}\right)-d_{i j}\left(t_{0}\right)=-d_{i j}\left(t_{0}\right)
$$

it follows:

$$
\begin{aligned}
& d_{i j}(t)=0 \quad \forall t>t_{0} \Longrightarrow q_{i j}(t)=0 \quad \forall t>t_{0} \\
& \Longrightarrow e_{i j} \notin E(t) \quad \forall t>t_{0} .
\end{aligned}
$$

\section{G. Proof of Lemma 8}

I) It follows directly from (9).

II) It follows directly from (5). In fact, since each Steiner vertex $v_{k} \in S \backslash\left\{v_{\sigma}\right\}$ acts as a sink for the protoplasmic flow induced by the Steiner vertex $v_{\sigma}$, and since there exists a unique path between $v_{k}$ and $v_{\sigma}$ in $\mathcal{T}$, then the protoplasmic flux $q_{i j}^{\sigma}$ flowing through $e_{i j}$ is proportional to the number of Steiner vertices connected with $v_{i}$ in the the subgraph $\left(V, E_{\mathcal{T}} \backslash\left\{e_{i j}\right\}\right)$.

III) From (11), if $q_{i j}^{\sigma}<0$, we have

$$
\begin{aligned}
q_{i j} & =\frac{1}{|S|} \sum_{v_{\sigma} \in S}\left|q_{i j}^{\sigma}\right| \\
& =\frac{1}{|S|}\left(\left(|S|-\left|S_{i j}^{\mathcal{T}}\right|\right) f_{0} \frac{\left|S_{i j}^{\mathcal{T}}\right|}{|S|-1}+\left|S_{i j}^{\mathcal{T}}\right| f_{0} \frac{|S|-\left|S_{i j}^{\mathcal{T}}\right|}{|S|-1}\right) .
\end{aligned}
$$

IV) It follows directly from (3), (11), and (12).

V) From (11), we have

$$
\begin{aligned}
L\left(E_{P_{i j}}\right)= & \sum_{e_{k_{l} k_{l+1}} \in E_{P_{i j}}} l_{k_{l} k_{l+1}} \\
= & \frac{1}{|S|} \sum_{e_{k_{l} k_{l+1}} \in E_{P_{i j}}} \sum_{v_{\sigma} \in S}\left|p_{k_{l}}^{\sigma}-p_{k_{l+1}}^{\sigma}\right| \\
= & \frac{1}{|S|} \sum_{v_{\sigma} \in S_{i k_{1}}^{\mathcal{T}} \bigcup \bigcup_{S^{\prime} k_{N-1}} \sum_{e_{k_{l} k_{l+1}} \in E_{P_{i j}}}\left|p_{k_{l}}^{\sigma}-p_{k_{l+1}}^{\sigma}\right|} \sum \frac{1}{|S|} \sum_{v_{\sigma} \notin S_{i k_{1}}^{\mathcal{T}} \bigcup S_{j k_{N-1}}^{\mathcal{T}}}\left|p_{k_{l}}^{\sigma}-p_{k_{l+1} k_{l+1}}^{\sigma}\right| . E_{P_{i j}} \mid
\end{aligned}
$$

We must consider now two cases: i) $v_{\sigma} \in S_{i k_{1}}^{\mathcal{T}} \cup S_{j k_{n-1}}^{\mathcal{T}}$; ii) $v_{\sigma} \notin S_{i k_{1}}^{\mathcal{T}} \bigcup S_{j k_{n-1}}^{\mathcal{T}}$.

$$
\begin{aligned}
\mathbf{p}^{\sigma T} \mathbf{A}^{\sigma} \mathbf{p}^{\sigma} & =\sum_{j=1, j \neq \sigma}^{n} p_{j}^{\sigma} \sum_{k=1, k \neq \sigma}^{n} a_{j k} p_{k}^{\sigma}=\sum_{j=1, j \neq \sigma}^{n} p_{j}^{\sigma} \sum_{k=1, k \neq j}^{n} \frac{d_{j k}}{l_{j k}}\left(p_{j}^{\sigma}-p_{k}^{\sigma}\right) \\
& =\sum_{j=1, j \neq \sigma}^{n}\left(\sum_{k=1, k \neq j, \sigma}^{n} \frac{d_{j k}}{l_{j k}} p_{j}^{\sigma 2}+\frac{d_{j \sigma}}{l_{j \sigma}} p_{j}^{\sigma 2}-\sum_{k=1, k \neq j, \sigma}^{n} \frac{d_{j k}}{l_{j k}} p_{j}^{\sigma} p_{k}^{\sigma}-\frac{d_{j \sigma}}{l_{j \sigma}} p_{j}^{\sigma} p_{\sigma}^{\sigma}\right) \\
& =\sum_{j=1, j \neq \sigma}^{n}\left(\sum_{k=1, k \neq \sigma}^{j-1} \frac{d_{j k}}{l_{j k}}\left(p_{j}^{\sigma 2}+p_{k}^{\sigma 2}\right)+\frac{d_{j \sigma}}{l_{j \sigma}} p_{j}^{\sigma 2}-\sum_{k=1, k \neq \sigma}^{j-1} 2 a_{j k} p_{j}^{\sigma} p_{k}^{\sigma}\right) \\
& =\sum_{j=1, j \neq \sigma}^{n}\left(\sum_{k=1, k \neq \sigma}^{j-1} \frac{d_{j k}}{l_{j k}}\left(p_{j}^{\sigma}-p_{k}^{\sigma}\right)^{2}+\frac{d_{j \sigma}}{l_{j \sigma}} p_{j}^{\sigma 2}\right) \geq 0
\end{aligned}
$$


Case i): Since from (4) either $\forall e_{k_{l} k_{l+1}} \in E_{P_{i j}}$ : $p_{k_{l}}^{\sigma}<p_{k_{l+1}}^{\sigma}$ or $\forall e_{k_{l} k_{l+1}} \in E_{P_{i j}}: p_{k_{l}}^{\sigma}>p_{k_{l+1}}^{\sigma}$, we have

$$
\begin{aligned}
& \frac{1}{|S|} \sum_{v_{\sigma} \in S_{i k_{1}}^{\mathcal{T}} \bigcup S_{S_{k_{N-1}}^{\tau}}} \sum_{e_{k_{l} k_{l+1}} \in P_{i j}}\left|p_{k_{l}}^{\sigma}-p_{k_{l+1}}^{\sigma}\right| \\
& =\frac{1}{|S|} \sum_{v_{\sigma} \in S_{i k_{1}}^{\mathcal{T}} \bigcup S_{j k_{N-1}}^{\mathcal{T}}}\left|p_{i}^{\sigma}-p_{j}^{\sigma}\right| .
\end{aligned}
$$

Case ii): Since from (4) it results $\forall e_{k_{l} k_{l+1}} \in$ $E_{\left.P_{i k(\sigma}\right)}: p_{k_{l}}^{\sigma}<p_{k_{l+1}}^{\sigma}$ and $\forall e_{k_{l} k_{l+1}} \in E_{P_{j k(\sigma)}}$ : $p_{k_{l}}^{\sigma}<p_{k_{l+1}}^{\sigma}$, we have

$$
\begin{aligned}
& \frac{1}{|S|} \sum_{v_{\sigma} \notin S_{i k_{1}}^{\mathcal{T}} \bigcup S_{S_{k_{N-1}}^{\mathcal{T}}}} \sum_{e_{k_{l} k_{l+1}} \in P_{i j}}\left|p_{k_{l}}^{\sigma}-p_{k_{l+1}}^{\sigma}\right| \\
& \quad=\frac{1}{|S|} \sum_{v_{\sigma} \notin S_{i k_{1}}^{\mathcal{T}} \bigcup S_{j_{k_{N-1}}}^{\mathcal{T}}}\left(\left|p_{i}^{\sigma}-p_{k(\sigma)}^{\sigma}\right|+\left|p_{j}^{\sigma}-p_{k(\sigma)}^{\sigma}\right|\right) .
\end{aligned}
$$

By substituting (34) and (35) in (33), we obtain the thesis (36), shown at the bottom of the page.

\section{H. Proof of Lemma 9}

We prove the lemma with a reductio ad absurdum by supposing that there exists an equilibrium point when $G(t)$ is not a tree and any cycle in $G(t)$ is a Physarum Cycle.

Since $G(t)$ is not a tree by hypothesis, we have that there exist cycles ${ }^{16}$ in $G(t)$. Without loss of generality, ${ }^{17}$ let us assume there exists a unique simple cycle $\mathcal{C}$ in $G(t)$.

We must consider two cases: i) $\left|S_{\mathcal{C}}\right|=0$; ii) $\left|S_{\mathcal{C}}\right|>0$.

Case i): At the equilibrium, from (15), we have

$$
\begin{aligned}
& L\left(E_{P_{i j}^{d, 2}}\right)=\frac{1}{|S|} \sum_{v_{\sigma} \in S}\left|p_{i}^{\sigma}-p_{j}^{\sigma}\right| \\
& L\left(E_{P_{i j}^{d, 1}}\right)=\frac{1}{|S|} \sum_{v_{\sigma} \in S}\left|p_{i}^{\sigma}-p_{j}^{\sigma}\right| .
\end{aligned}
$$

${ }^{16} \mathrm{~A}$ tree is a connected graph with no cycles. Since, from Theorem $1, G(t)$ is connected, then we have cycles in $G(t)$

${ }^{17}$ If the assumption does not hold, the lemma can be easily proved by adopting the same reasoning.
Clearly, (37) and (38) constitute a reductio ad absurdum since $L\left(E_{P_{i j}^{d, 2}}\right)>L\left(E_{P_{i j}^{d, 1}}\right)$ for reductio's hypothesis.

Case ii): At the equilibrium, by considering the direct path $P_{k l}^{d}$, from (15), we have

$$
\frac{1}{|S|} \sum_{v_{\sigma} \in S}\left|p_{k}^{\sigma}-p_{l}^{\sigma}\right|=L\left(E_{P_{k l}^{d}}\right) .
$$

Moreover, by considering the indirect path $P_{k l}^{i}$, from (14), we have (40), shown at the bottom of the page.

Clearly, (39) and (40) constitute a reductio ad absurdum for the reductio's hypothesis.

\section{Proof of Lemma 10}

Since $G(t)$ is not a tree by hypothesis, we have that there exist cycles in $G(t)$. Without loss of generality, ${ }^{18}$ let us assume there exists a unique simple cycle $\mathcal{C}$ in $G(t)$. Consequently, from Theorem 1, we have that there exist two Steiner trees in $G(t)$.

Let $P_{\text {opt }}=\mathcal{C} \cap \mathcal{T}_{\text {opt }}$ denote the path belonging to the minimum-length Steiner tree $\mathcal{T}_{\text {opt }}$, with $E_{P_{\text {opt }}}=\left\{e_{i_{k} i_{k+1}}\right\}_{k=0}^{n-1}$. Similarly, let $P=\mathcal{C} \backslash P^{\text {opt }}$ denote the path in $G(t) \backslash \mathcal{T}_{\text {opt }}$, with $E_{P}=\left\{e_{j_{k} j_{k+1}}\right\}_{k=0}^{m-1}$. Since $\mathcal{C}$ is a cycle, we have $i_{0}=j_{0}$ and $i_{n}=j_{m}$.

We define the function $V: \mathbf{D}(t) \longrightarrow \mathcal{R}_{0}^{+}$as

$$
\begin{aligned}
& V(\mathbf{D}(t)) \\
& =\ln W(\mathbf{D}(t)) \\
& =\ln \frac{\prod_{e_{i j} \in E_{P}} d_{i j}^{l_{i j}}(t)}{\prod_{e_{i j} \in E_{P_{\mathrm{opt}}}} d_{i j}^{l_{i j}}(t)}=\ln \frac{\prod_{k=0}^{m-1} d_{j_{k} j_{k+1}}^{l_{j_{k} j_{k+1}}}(t)}{\prod_{k=0}^{n-1} d_{i_{k} i_{k+1}}^{l_{k} i_{k+1}}(t)} \\
& =\sum_{k=0}^{m-1} l_{j_{k} j_{k+1}} \ln d_{j_{k} j_{k+1}}(t)-\sum_{k=0}^{n-1} l_{i_{k} i_{k+1}} \ln d_{i_{k} i_{k+1}}(t) .
\end{aligned}
$$

Since

$$
\begin{aligned}
\frac{d}{d t}\left(l_{i j} \ln d_{i j}(t)\right) & =\frac{l_{i j}}{d_{i j}(t)} \dot{d}_{i j}(t)=\frac{l_{i j}}{d_{i j}(t)}\left(q_{i j}(t)-d_{i j}(t)\right) \\
& =\frac{1}{|S|} \sum_{v_{\sigma} \in S}\left|p_{i}^{\sigma}(t)-p_{j}^{\sigma}(t)\right|-l_{i j}
\end{aligned}
$$

we have (43), shown at the top of the next page.

${ }^{18}$ If the assumption does not hold, the lemma can be easily proved by adopting the same reasoning.

$$
L\left(E\left(P_{i j}\right)\right)=\frac{1}{|S|} \sum_{v_{\sigma} \in S}\left|p_{i}^{\sigma}+p_{j}^{\sigma}\right|-\frac{1}{|S|} \cdot \sum_{v_{\sigma} \notin S_{i k_{1}}^{\tau}} \bigcup_{S_{j k_{N-1}}^{\mathcal{T}}}\left(\left|p_{i}^{\sigma}-p_{k(\sigma)}^{\sigma}\right|+\left|p_{j}^{\sigma}-p_{k(\sigma)}^{\sigma}\right|-\left|p_{i}^{\sigma}-p_{j}^{\sigma}\right|\right) .
$$






$$
\begin{aligned}
\dot{V} \mathbf{D}(t)) & =\sum_{k=0}^{m-1}\left(\frac{1}{|S|} \sum_{v_{\sigma} \in S}\left|p_{j_{k}}^{\sigma}(t)-p_{j_{k+1}}^{\sigma}(t)\right|-l_{j_{k} j_{k+1}}\right)-\sum_{k=0}^{n-1}\left(\frac{1}{|S|} \sum_{v_{\sigma} \in S}\left|p_{i_{k}}^{\sigma}(t)-p_{i_{k+1}}^{\sigma}(t)\right|-l_{i_{k} i_{k+1}}\right) \\
& =\frac{1}{|S|} \sum_{v_{\sigma} \in S}\left(\sum_{k=0}^{m-1}\left|p_{j_{k}}^{\sigma}(t)-p_{j_{k+1}}^{\sigma}(t)\right|-\sum_{k=0}^{n-1}\left|p_{i_{k}}^{\sigma}(t)-p_{i_{k+1}}^{\sigma}(t)\right|\right)+L\left(E_{P_{\mathrm{opt}}}\right)-L\left(E_{P}\right) .
\end{aligned}
$$

$$
\dot{V}(\mathbf{D}(t))=\frac{1}{|S|} \sum_{v_{\sigma} \in S}\left(\left|p_{j_{0}}^{\sigma}(t)-p_{j_{m}}^{\sigma}(t)\right|-\left|p_{i_{0}}^{\sigma}(t)-p_{i_{m}}^{\sigma}(t)\right|\right)+L\left(E_{P_{\mathrm{opt}}}\right)-L\left(E_{P}\right)=L\left(E_{P_{\mathrm{opt}}}\right)-L\left(E_{P}\right)<0
$$

$$
\dot{V}(\mathbf{D}(t))=\frac{1}{|S|} \sum_{\substack{v_{\sigma} \notin S_{i_{0} i_{1}}^{\mathcal{T}_{\mathrm{ot}}} \bigcup \\ S_{i_{n} i_{n}-1}^{\mathcal{T}_{\mathrm{opt}}}}}\left(\sum_{k=0}^{m-1}\left|p_{j_{k}}^{\sigma}(t)-p_{j_{k+1}}^{\sigma}(t)\right|-\sum_{k=0}^{n-1}\left|p_{i_{k}}^{\sigma}(t)-p_{i_{k+1}}^{\sigma}(t)\right|\right)+L\left(E_{P_{\mathrm{opt}}}\right)-L\left(E_{P}\right) .
$$

$$
\dot{V}(\mathbf{D}(t))=\frac{1}{|S|} \sum_{\substack{v_{\sigma} \notin S_{i_{0} i_{1}}^{\tau_{\mathrm{opt}}}} S_{i_{n} i_{n}-1}^{\tau_{\text {opt }}}}\left(\left|p_{j_{0}}^{\sigma}(t)-p_{k(\sigma)}^{\sigma}(t)\right|+\left|p_{j_{m}}^{\sigma}(t)-p_{k(\sigma)}^{\sigma}(t)\right|-\left|p_{i_{0}}^{\sigma}(t)-p_{i_{n}}^{\sigma}(t)\right|\right)+L\left(E_{P_{\mathrm{opt}}}\right)-L\left(E_{P}\right)<0
$$

We must consider two cases: i) $\left|S_{\mathcal{C}}\right|=0$; ii) $\left|S_{\mathcal{C}}\right|>0$.

i) From (4), for any $v_{\sigma} \in S$, it results either $p_{i_{k}}^{\sigma}<p_{i_{k+1}}^{\sigma} \wedge$ $p_{j_{k}}^{\sigma}<p_{j_{k+1}}^{\sigma} \forall k$ or $p_{i_{k}}^{\sigma}>p_{i_{k+1}}^{\sigma} \wedge p_{j_{k}}^{\sigma}>p_{j_{k+1}}^{\sigma} \forall k$. Thus, we have (44), shown at the top of the page.

ii) From (4), for any $v_{\sigma} \in S_{i_{0} i_{1}}^{\mathcal{T}_{\text {ot }}} \bigcup S_{i_{n} i_{n}-1}^{\mathcal{T}_{\text {ot }}}$, we have either $p_{i_{k}}^{\sigma}<p_{i_{k+1}}^{\sigma} \wedge p_{j_{k}}^{\sigma}<p_{j_{k+1}}^{\sigma} \forall k$ or $p_{i_{k}}^{\sigma}>p_{i_{k+1}}^{\sigma} \wedge p_{j_{k}}^{\sigma}>$ $p_{j_{k+1}}^{\sigma} \forall k$. Thus, following the same reasoning of (44), we have (45) shown at the top of the page.

Finally, by noting that $P_{\mathrm{opt}}=P_{k l}^{i}$ and $P=P_{k l}^{d}$ as in (17) and (18), we obtain (46), shown at the top of the page, where the inequality holds since $\mathcal{C}$ is a Physarum cycle by hypothesis.

Let us now discuss function $W(\mathbf{D}(t))$ defined in (41). We first observe that

$$
\begin{aligned}
W(\mathbf{D}(t)) \geq 0 & \forall \mathbf{D}(t) \in H \\
W(\mathbf{D}(t))=0 & \Longleftrightarrow \exists e_{i j} \in E_{P}: d_{i j}(t)=0 \\
& \Longrightarrow d_{i j}(t)=0 \quad \forall e_{i j} \in E_{P}
\end{aligned}
$$

where the last implication follows from (4). Moreover, by accounting for (44) and (44), we have

$$
\begin{aligned}
\dot{W}(\mathbf{D}(t)) & =W(\mathbf{D}(t)) \dot{V}(\mathbf{D}(t)) \\
& =\left(L\left(E_{P_{\text {opt }}}\right)-L\left(E_{P}\right)\right) W(\mathbf{D}(t))<W(\mathbf{D}(t))
\end{aligned}
$$

and

$$
\begin{aligned}
\dot{W}(\mathbf{D}(t))=0 & \Longleftrightarrow \exists e_{i j} \in E_{P}: d_{i j}(t)=0 \\
& \Longrightarrow d_{i j}(t)=0 \quad \forall e_{i j} \in E_{P} .
\end{aligned}
$$

Consequently, since $W(\mathbf{D}(t))$ is positive in $H$ and it exponentially tends to zero as $t$ increases, and by accounting for Lemma 9, we have that there exists $e_{i j} \in E_{P}=E(t)-E_{\mathcal{T}_{\mathrm{opt}}}$ so that $d_{i j}(t)$ exponentially tends to zero as $t$ increases.

\section{ACKNOWLEDGMENT}

The authors would like to thank Dr. A. S. Cacciapuoti, Dr. J. M. Jornet, Dr. M. Pierobon, and Dr. P. Wang for their valuable feedback.

\section{REFERENCES}

[1] F. Dressler and O. B. Akan, "A survey on bio-inspired networking," Comput. Netw., vol. 54, no. 6, pp. 881-900, 2010.

[2] S. Balasubramaniam, K. Leibnitz, P. Lio, D. Botvich, and M. Murata, "Biological principles for future Internet architecture design," IEEE Commun. Mag., vol. 49, no. 7, pp. 44-52, Jul. 2011.

[3] T. Nakagaki, H. Yamada, and A. Toth, "Intelligence: Maze-solving by an amoeboid organism," Nature, vol. 407, no. 6803, p. 470, 2000.

[4] A. Adamatzky, V. Erokhin, M. Grube, T. Schubert, and A. Schumann, "Physarum chip project: Growing computers from slime mould," Int. J. Unconvent. Comput., vol. 8, no. 4, pp. 319-323, 2012.

[5] "Physarum chip: Growing computers from slime mould," Funded by the Seventh Framework Programme (FP7) by the European Commission within CORDIS and the FET Proactive scheme, Mar. 2013.

[6] T. Nakagaki, H. Yamada, and M. Hara, "Smart network solutions in an amoeboid organism," Biophys. Chem., vol. 107, no. 1, pp. 1-5, 2004

[7] A. Tero, R. Kobayashi, and T. Nakagaki, "Physarum solver: A biologically inspired method of road-network navigation," Physica A, Statist. Mech. Appl., vol. 363, no. 1, pp. 115-119, 2006.

[8] A. Tero, R. Kobayashi, and T. Nakagaki, "A mathematical model for adaptive transport network in path finding by true slime mold," $J$. Theoret. Biol., vol. 244, no. 4, pp. 553-564, 2007.

[9] T. Miyaji and I. Ohnishi, "Physarum can solve the shortest path problem on Riemannian surface mathematically rigorously," Int. J. Pure Appl. Math., vol. 47, no. 3, pp. 353-369, 2008. 
[10] A. Tero, T. Nakagaki, K. Toyabe, K. Yumiki, and R. Kobayashi, "A method inspired by Physarum for solving the Steiner problem," Int. $J$. Unconvent. Comput., vol. 6, no. 1, pp. 109-123, 2010.

[11] S. Tsuda, K.-P. Zauner, and Y.-P. Gunji, "Robot control with biological cells," Biosystems, vol. 87, no. 2-3, pp. 215-223, 2007.

[12] F. K. Hwang, D. S. Richards, and P. Winter, The Steiner Tree Problem, ser. Annals of Discrete Mathematics. Philadelphia, PA, USA: SIAM, 1992, vol. 53

[13] R. M. Karp, "Reducibility among combinatorial problems," in Complexity of Computer Computations. New York, NY, USA: Plenum, 1972, pp. 85-103.

[14] A. Nakamura and K. Kohama, "Calcium regulation of the actin-myosin interaction of Physarum polycephalum," in A Survey of Cell Biology. New York, NY, USA: Academic, 1999, vol. 191, pp. 53-98.

[15] H. T. Siegelmann and S. Fishman, "Analog computation with dynamical systems," Physica D, Nonlinear Phenomena, vol. 120, no. 1-2, pp. 214-235, 1998.

[16] H. T. Siegelmann, A. Ben-Hur, and S. Fishman, "Computational complexity for continuous time dynamics," Phys. Rev. Lett., vol. 83, pp. 1463-1466, 1999.

[17] L. Kou, G. Markowsky, and L. Berman, "A fast algorithm for Steiner trees," Acta Inf., vol. 15, no. 2, pp. 141-145, 1981.

[18] T. Koch, A. Martin, and S. Voß, "SteinLib: An updated library on Steiner Tree problems in graphs," Konrad-Zuse-Zentrum für Informationstechnik Berlin, Berlin, Germany, Tech. Rep. ZIB-Report 00-37, 2000.

[19] R. A. Horn and C. R. Johnson, Matrix Analysis. Cambridge, U.K.: Cambridge Univ. Press, 1990.



Marcello Caleffi (M'11) received the Dr. Eng. degree (summa cum laude) in computer science engineering from the University of Lecce, Lecce, Italy, in 2005, and the Ph.D. degree in electronic and telecommunications engineering from the University of Naples Federico II, Naples, Italy, in 2009.

Currently, he is an Assistant Professor with the Department of Electrical Engineering and Information Technologies, University of Naples Federico II. From 2010 to 2011, he was with the Broadband Wireless Networking Laboratory, Georgia Institute of Technology, Atlanta, GA, USA, as a Visiting Researcher. In 2011, he was also with the NaNoNetworking Center in Catalunya (N3Cat), Universitat Politècnica de Catalunya (UPC), Barcelona, Spain, as a Visiting Researcher. His research interests are in cognitive radio networks and biological networks.

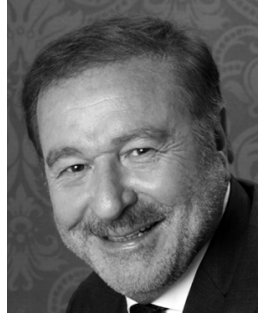

Ian F. Akyildiz (M'86-SM'89-F'96) received the B.S., M.S., and Ph.D. degrees in computer engineering from the University of Erlangen-Nürnberg, Nuremberg, Germany, in 1978, 1981, and 1984 respectively.

Currently, he is the Ken Byers Chair Professor in Telecommunications with the School of Electrical and Computer Engineering, Georgia Institute of Technology (Georgia Tech), Atlanta, GA, USA, as well as the Director of the Broadband Wireless Networking Laboratory and Chair of the Telecommunication Group at Georgia Tech. He is an Honorary Professor with the School of Electrical Engineering, Universitat Politècnica de Catalunya (UPC), Barcelona, Spain, and the founder of the NaNoNetworking Center in Catalunya (N3Cat). Since 2011, he has been a Consulting Chair Professor with the Department of Information Technology, King Abdulaziz University (KAU), Jeddah, Saudi Arabia. Since 2013, he has also been a Finland Distinguished Professor Program (FiDiPro, supported by the Academy of Finland) Professor with the Department of Communications Engineering, Tampere University of Technology, Tampere, Finland. His current research interests are in nanonetworks, Long Term Evolution (LTE) advanced networks, cognitive radio networks, and wireless sensor networks.

Dr. Akyildiz has been an Association for Computing Machinery (ACM) Fellow since 1997. He received numerous awards from the IEEE and ACM. He is the Editor-in-Chief of Computer Networks and the founding Editor-in-Chief of Ad Hoc Networks, Physical Communication, and Nano Communication Networks.

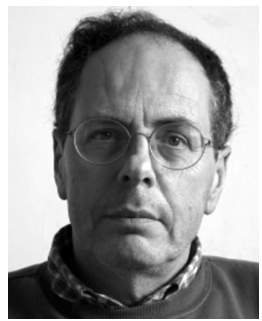

Luigi Paura (M'11) received the Dr. Eng. degree (summa cum laude) in electronic engineering from the University of Napoli Federico II, Naples, Italy, in 1974.

From 1979 to 1984, he was with the Department of Biomedical, Electronic and Telecommunications Engineering, University of Naples Federico II, first as an Assistant Professor and then as an Associate Professor. Since 1994, he has been a Full Professor of telecommunications: first, with the Department of Mathematics, University of Lecce, Lecce, Italy; then, with the Department of Information Engineering, Second University of Naples, Naples, Italy; and, finally, since 1998 he has been with the Department of Biomedical, Electronic and Telecommunications Engineering, University of Naples Federico II. He also held teaching positions with the University of Salerno, Salerno, Italy; University of Sannio, Benevento, Italy; and University Parthenope of Naples, Naples, Italy. In 1985 to 1986 and in 1991, he was a Visiting Researcher with the Signal and Image Processing Lab, University of California, Davis, CA, USA. His research interests are mainly in digital communication systems and cognitive radio networks. 